EPJ manuscript No.

(will be inserted by the editor)

\title{
Exact results for the Kardar-Parisi-Zhang equation with spatially correlated noise
}

\author{
H. K. Janssen ${ }^{1}$, U.C. Täuber ${ }^{2}$, and E. Frey ${ }^{2}$ \\ 1 Institut für Theoretische Physik III, Heinrich-Heine-Universität, 40225 Düsseldorf, Germany \\ e-mail: janssen@thphy.uni-duesseldorf.de \\ 2 Institut für Theoretische Physik T34,Technische Universität München, 85747 Garching, Germany \\ e-mail: taeuber@physik.tu-muenchen.de,frey@physik.tu-muenchen.de
}

March 2, 2022

Dedicated to Franz Schwabl on the occasion of his 60th birthday.

\begin{abstract}
We investigate the Kardar-Parisi-Zhang (KPZ) equation in $d$ spatial dimensions with Gaussian spatially long-range correlated noise - characterized by its second moment $R\left(\mathbf{x}-\mathbf{x}^{\prime}\right) \propto$ $\left|\mathbf{x}-\mathbf{x}^{\prime}\right|^{2 \rho-d}$ — by means of dynamic field theory and the renormalization group. Using a stochastic Cole-Hopf transformation we derive exact exponents and scaling functions for the roughening transition and the smooth phase above the lower critical dimension $d_{c}=2(1+\rho)$. Below the lower critical dimension, there is a line $\rho_{*}(d)$ marking the stability boundary between the short-range and long-range noise fixed points. For $\rho \geq \rho_{*}(d)$, the general structure of the renormalization-group equations fixes the values of the dynamic and roughness exponents exactly, whereas above $\rho_{*}(d)$, one has to rely on some perturbational techniques. We discuss the location of this stability boundary $\rho_{*}(d)$ in light of the exact results derived in this paper, and from results known in the literature. In particular, we conjecture that there might be two qualitatively different strong-coupling phases above and below the lower critical dimension, respectively.
\end{abstract}

PACS. 64.60.Ht Dynamic critical phenomena - 64.60.Ak Renormalization-group, fractal, and percolation studies of phase transitions $-05.40 .+\mathrm{j}$ Fluctuation phenomena, random processes, and Brownian motion - 05.70.Ln Non-equilibrium thermodynamics, irreversible processes

\section{Introduction}

The dynamics of interfaces moving through random media has turned out to be one of the most fascinating and at the same time challenging topics in theoretical nonequilibrium physics. Among many other problems, kinetic roughening of growing interfaces comprises one interesting class of such phenomena. Here the randomness is described by an annealed random noise which mimics the random adsorption of molecules onto a surface. The average force on the interface is unimportant and may be removed from the equations of motion. Theoretical modeling of those growth processes started with the work by Edwards and Wilkinson (EW) [1] who suggested that one might describe the dynamics of the height fluctuations by a simple linear diffusion equation. Kardar, Parisi, and Zhang (KPZ) 2 realized that there is a relevant term proportional to the square of the height gradient which represents a correction for lateral growth. What at first glance seemed to be a simple generalization of the diffusion equation became an archetypical example of non-equilibrium dynamic phenomena [3, [4, 5].
Despite its fundamental importance as a generic theoretical model for non-equilibrium phase transitions, to date there seems to be no experimental system which quantitatively confirms the predictions of the KPZ model satisfactorily [4,5]. This discrepancy has inspired the introduction of various modifications of the original model. There are several ways in which the KPZ equation provides an incomplete description of an actual experimental system: First of all, one might question the validity of assuming uncorrelated Gaussian noise. In a real system, the noise could be correlated [6], non-Gaussian [7], or even quenched [8.9] instead of annealed. Next, in many physical processes there exist long-range hydrodynamic interactions leading to non-local dynamics for the height fluctuations 10.11. Finally, there may be other non-linearities which describe important physics omitted in the KPZ equation, in particular in the strong-coupling rough phase.

Quite generally, in a genuine non-equilibrium system, the form of the noise correlations in an effective Langevintype description is a crucial ingredient of the modeling. This is in contrast to equilibrium dynamics, where the 
H.K. Janssen et al.: Exact results for the KPZ equation with spatially correlated noise

functional form of the noise as well as its strength are fixed via an Einstein relation (detailed-balance condition) which ensures that asymptotically the probability distribution will be the equilibrium one. Uncorrelated white noise is often a straightforward choice, but the sensitivity and stability of the ensuing results need to be carefully tested against modifications of the noise correlations. In terms of the renormalization-group (RG) language, one must establish which modifications of the noise correlators constitute relevant perturbations, and may thus lead to novel universality classes.

In this work, we focus on the effect of spatially correlated Gaussian noise $\tilde{\zeta}(\mathbf{x}, t)$ with

$$
\left\langle\tilde{\zeta}(\mathbf{x}, t) \tilde{\zeta}\left(\mathbf{x}^{\prime}, t^{\prime}\right)\right\rangle=2 R\left(\mathbf{x}-\mathbf{x}^{\prime}\right) \delta\left(t-t^{\prime}\right)
$$

where for some part of the paper we will restrict ourselves to the particular form

$$
R\left(\mathbf{x}-\mathbf{x}^{\prime}\right) \propto\left|\mathbf{x}-\mathbf{x}^{\prime}\right|^{2 \rho-d}
$$

with $d$ being the spatial dimension of the surface. Our motivation, however, is not to introduce a realistic growth model which allows for a quantitative comparison with experiments, but to gain a better understanding of the KPZ equation and possibly reveal some of its hidden secrets. Since noise correlations are actually generated by the non-linearity in the KPZ equation, one might hope that via introducing such correlations to begin with, one could learn something about the notorious strong-coupling behavior of the rough phase, separated from the smooth EW regime through a second-order dynamic roughening transition (above the lower critical dimension $d_{c}$ ).

The KPZ equation with correlated Gaussian noise has first been studied by Medina et al. [6], applying dynamic renormalization-group (RG) methods. Among other results, they found in a one-loop perturbative renormalization-group analysis that for the $(1+1)$-dimensional growth model, there exists a critical value of the correlation exponent, $\rho_{c}=\frac{1}{4}$, below which long-range noise correlations are irrelevant and the roughness and dynamic critical exponents retain their values $\chi=\frac{1}{2}$ and $z=\frac{3}{2}$ associated with uncorrelated (white) noise. Above $\rho_{c}$, long-range noise correlations become relevant in the RG sense, and a novel fixed point emerges, at which the exponents assume the values

$$
\chi=\frac{1+2 \rho}{3}, \quad \text { and } \quad z=\frac{5-2 \rho}{3} .
$$

These results have actually been found already by Kardar [12] and Nattermann [13] in the context of a generalized interface model for domain wall roughening which interpolates between a random bond $(\rho=0)$ and random field $(\rho=1)$ Ising model; some limiting results were also obtained by Zhang [14 building on Ref. [15]. Eq. 1.3 also corresponds to an estimate for the exponents obtained by Flory scaling [12. In addition, these results are in accord with a functional renormalization-group calculation by Halpin-Healy [16,17. The above values are, however, in conflict with results obtained by means of a replica scaling method [18], which give critical exponents quite different from (1.3), and would predict a critical value of $\rho_{c}=\frac{1}{2}$.

To date, there have also been several numerical investigations to determine the dependence of the critical exponents on the correlation exponent of the noise $\rho$. For $d=1$, early simulations 19 using restricted solid-onsolid (RSOS) and ballistic deposition models, seemed to strongly support the predictions of the dynamic RG calculation with $\rho_{c}=\frac{1}{4}$. These results, however, could not be confirmed by later simulations using complementary models 20. Employing an alternative scheme to generate correlated noise, Pang et al. 21] carried out extensive numerical studies on the RSOS model and the presumably equivalent model of a directed polymer in a random medium (DPRM). It seems that the exponents obtained from the simulation of the RSOS model deviate from the results for the short-range fixed point already at $\rho \approx \frac{1}{8}$; the DPRM data break off even earlier. In summary, there is no coherent picture emerging from the numerical simulations performed on the basis of an interface-height or directed-polymer representation, respectively. We suppose that these discrepancies between different simulations may partly be traced back to the presence of corrections-toscaling effects due to the crossover between the shortrange and long-range fixed point. Our impression is that a careful re-analysis of the numerical simulations in the light of the results presented in this paper would be highly desirable.

Hayot and Jayaprakash [22,23] have recently investigated the effect of correlated noise in the framework of the stochastic Burgers equation. They find very clear evidence that the critical value for $\rho$ is $\rho_{c}=\frac{1}{4}$, and that for $\frac{1}{4} \leq \rho \leq 1$ the asymptotic behavior is within numerical errors described by the exponents derived from the one-loop RG calculation, (1.3). The most surprising and remarkable result of this paper is that the numerical simulations show quantitative agreement with the one-loop results for the roughness exponent $\chi$ even for $\rho \geq 1$, i.e., in a parameter regime which (because of higher-order nonlinear terms) lies outside the realm of the RG analysis. At these large values of $\rho>1$, the velocity fields $u(x, t)$ in the statistically stationary state display well-defined shocks leading to multifractality and anomalous dynamics. The truncated velocity correlation function $\left\langle[u(x, t)-u(x, 0)]^{2}\right\rangle$ displays two distinct scaling regimes. At short times, the dynamic exponent $z$ is given by the one-loop result (1.3), while $z \approx 1$ at longer times. The value $z=1$ is a consequence of the presence and ballistic motion of the shock fronts. Through an analysis of the scaling behavior of appropriate composite operators, it is found that these higher-order operators do not become increasingly relevant. This suggests the validity of simple balancing arguments 22, which lead to exactly the same exponent relations (1.3) as the one-loop result obtained by Medina et al. [6].

Furthermore, the $(2+1)$-dimensional KPZ model was investigated by direct numerical integration of the KPZ equation [24. Similar to the case $d=1$, there seems to be a critical value $\rho_{c} \approx 0.5$ below which the exponents for 
uncorrelated noise are oberved, and above which a strong $\rho$-dependence of the critical exponents is found. Since a one-loop approximation [6] does not lead to a stable fixed point in two dimensions, a comparison with analytic results was not possible up to now. We will see later in this paper that our analysis not only allows for such a comparison, but even leads to exact expressions for the critical exponents (provided the associated finite RG fixed points exist).

The outline of the paper is as follows. In the subsequent section, we set up the field-theoretical tools (such as the dynamic functional, renormalization factors, renormalization-group equations, and scaling relations) for the analysis of the KPZ equation with correlated noise. This section contains mostly definitions and recapitulations, as well as discussions of results known in the literature [6] in light of the field-theory framework. In particular, we present a scrutinized discussion of the derivation of the dynamic functional from the stochastic equation of motion, which pays attention to some subtleties often neglected in the literature, yet of considerable importance for the stochastic Cole-Hopf transformation to be introduced in section 4.1. Section 3 then deals with an analysis of the critical behavior below the lower critical dimension $d_{c}=2(1+\rho)$ where there is only a rough phase, and no roughening transition from a smooth into a rough phase exists. Since within a formulation in terms of the height variables $h(\mathbf{x}, t)$, there is no critical dimension around which one could try to perform an $\varepsilon$ expansion, we have to rely on a fixed-dimension loop expansion which necessarily becomes non-minimal in the sense that UV-finite terms in the renormalization constants need to be retained. Notice, however, that there is one special point in the $(\rho, d)$-plane, corresponding to the Burgers equation with non-onserved noise, which actually allows for an $\varepsilon$ expansion around the upper critical dimension 4 [15]. Here we show how one may perform a fixed-dimension expansion below $d_{c}$, the applicability of which, however, seems mainly be restricted to the $(1+1)$ dimensional situation, where the critical behavior can also be analyzed by starting from a driven diffusion equation.

The behavior of the roughening transition above the critical dimension $d_{c}$ is studied in section 4 by means of a stochastic Cole-Hopf transformation, which transforms the dynamic functional of the KPZ equation into a dynamic functional whose structure is very reminiscent of the field theory for diffusion-limited pair annihilation [25.26. 27]. This allows us to present exact expressions for the entire set of renormalization-group flow functions, in particular the RG beta functions. We find that the roughening transition in the presence of correlated noise is characterized by the dynamic exponent $z_{c}=2$, the roughness exponent $\chi_{c}=0$, the crossover exponent $\phi_{c}=d-2$, and the correction-to-scaling exponent $\omega_{c}=d-2(1+\rho)$. Above the lower critical dimension $d_{c}$, the long-range scaling fixed point is stable, and the smooth phase is described by the scaling exponents $z_{\mathrm{sm}}=2, \chi_{\mathrm{sm}}=1+\rho-\frac{d}{2}$, and the different correction-to-scaling exponents $\omega_{1}=d-2(1+\rho)$, $\omega_{2}=2(1+2 \rho)-d$ for $2(1+\rho)<d<2(1+2 \rho)$, and $\omega_{1}=d-2(1+2 \rho), \omega_{2}=2 \rho$ for $d \geq 2(1+2 \rho)$, respectively.
The rough phase is found to be non-accessible through perturbational means. Finally, for $d>d_{c}$ the theory can be generalized to an arbitrary noise correlator (albeit local in time). The ensuing Bethe-Salpeter integral equation is analyzed numerically for Gaussian and power-law noise spectrum in section 5 . We conclude with a brief summary, and a discussion of some of the open problems.

\section{The KPZ field theory with spatially correlated noise}

\subsection{Model equations and field theory}

In the standard convention, the Kardar-Parisi-Zhang or KPZ equation for the interface height fluctuation $h(\mathbf{x}, t)$ in $d+1$ dimensions is written in the form [2, 6]

$$
\frac{\partial h}{\partial t}=D \nabla^{2} h+\frac{\lambda}{2}(\nabla h)^{2}+\tilde{\zeta},
$$

where $D$ denotes the effective interface surface tension, or, in the Burgers representation, the kinematic viscosity of the potential velocity field $\mathbf{u} \propto \nabla h$. The coefficient $\lambda$ describes the strength of the non-linearity, associated with either the curvature-driven growth process or the convective term in the hydrodynamic equation, respectively.

Furthermore, $\tilde{\zeta}$ denotes a stochastic driving force with zero mean, $\langle\tilde{\zeta}(\mathbf{x}, t)\rangle$, and Gaussian correlations determined by the second moment

$$
\left\langle\tilde{\zeta}(\mathbf{x}, t) \tilde{\zeta}\left(\mathbf{x}^{\prime}, t^{\prime}\right)\right\rangle=2 \widetilde{R}\left(\mathbf{x}-\mathbf{x}^{\prime}\right) \delta\left(t-t^{\prime}\right),
$$

which we assume to be local in time but long-range correlated in space. In particular, we are going to consider the case where in Fourier representation

$$
\widetilde{R}(\mathbf{x})=\int \frac{d^{q} q}{(2 \pi)^{d}} \widetilde{R}(\mathbf{q}) \exp (i \mathbf{q} \cdot \mathbf{x})
$$

takes the form

$$
\widetilde{R}(\mathbf{q})=\widetilde{D}\left(1+w q^{-2 \rho}\right) .
$$

Here, in addition to the short-range term proportional to $\widetilde{D}$, we have allowed for a power-law long-range contribution to $\widetilde{R}(\mathbf{x}) \propto x^{2 \rho-d}$ with amplitude $\widetilde{\Delta}=\widetilde{D} w[6]$.

For the field-theoretic analysis, and a direct transfer of standard results from equilibrium critical dynamics to the analysis of the above non-equilibrium Langevin equation, it is more appropriate to rescale the fluctuating and noise fields according to $s(\mathbf{x}, t)=(D / \widetilde{D})^{\frac{1}{2}} h(\mathbf{x}, t)$ and $\zeta(\mathbf{x}, t)=$ $(D / \widetilde{D})^{\frac{1}{2}} \tilde{\zeta}(\mathbf{x}, t)$, which leads to

$$
\frac{\partial s}{\partial t}=D \nabla^{2} s+\frac{D g}{2}(\nabla s)^{2}+\zeta,
$$

where $g=\lambda\left(\widetilde{D} / D^{3}\right)^{\frac{1}{2}}$ represents the effective non-linear coupling of the KPZ problem. In this reparametrisation, the noise correlations in Fourier space read

$$
\begin{aligned}
&\left\langle\zeta(\mathbf{q}, \omega) \zeta\left(\mathbf{q}^{\prime}, \omega^{\prime}\right)\right\rangle=2 D\left(1+w q^{-2 \rho}\right) \\
& \times(2 \pi)^{d} \delta\left(\mathbf{q}+\mathbf{q}^{\prime}\right) \delta\left(\omega+\omega^{\prime}\right),
\end{aligned}
$$


with $w$ characterizing the relative strength of the spatially long-range and local noise correlations, respectively. Notice that through this simple rescaling, we have rendered the local-noise amplitude identical with the kinetic coefficient in the Langevin equation. However, this does not in general imply, even for $w=0$, that detailed balance holds. For, despite the validity of the Einstein relation for $w=0$, the integrability condition 28 for the reversible force term $D g(\nabla S)^{2} / 2$ in Eq. (2.5) is fulfilled in one dimension only. It is well known that in this case the stationary probability distribution is given by

$$
\mathcal{P}_{s t}[s] \propto \exp (-\mathcal{H}[s])=\exp \left(-\frac{1}{2} \int_{x}(\nabla s)^{2}\right),
$$

where we have introduced the short-hand notation $\int_{x}=$ $\int d^{d} x$.

Langevin equations like the KPZ equation (2.5) are not well-suited for the development of a systematic renormalized field theory. Furthermore, their interpretation depends on the underlying time discretization. Here, we shall use an interpretation in the sense of Ito. Instead of the Langevin equation, we write down the corresponding Gaussian transition probability distribution $\mathcal{P}[s(t+\tau) \mid s(t)]$ from a configuration $\{s(\mathbf{x}, t)\}$ at time $t$ to a configuration $\{s(\mathbf{x}, t+\tau)\}$ at time $t+\tau$, where $\tau$ is a small time increment,

$$
\begin{aligned}
& \mathcal{P}[s(t+\tau) \mid s(t)] \propto \\
& \exp \left(-\frac{1}{4 \tau} \int_{x, x^{\prime}}[s(\mathbf{x}, t+\tau)-s(\mathbf{x}, t)-\tau V(s(\mathbf{x}, t))]\right. \\
& \left.\times R^{-1}\left(\mathbf{x}-\mathbf{x}^{\prime}\right)\left[s\left(\mathbf{x}^{\prime}, t+\tau\right)-s\left(\mathbf{x}^{\prime}, t\right)-\tau V\left(s\left(\mathbf{x}^{\prime}, t\right)\right)\right]\right) \\
& =\int \mathcal{D}[\tilde{s}(t+\tau)] \exp \left(-\int_{x} \tilde{s}(\mathbf{x}, t+\tau)[s(\mathbf{x}, t+\tau)\right. \\
& \quad-s(\mathbf{x}, t)-\tau V(s(\mathbf{x}, t))] \\
& \left.+\tau \int_{x, x^{\prime}} \tilde{s}(\mathbf{x}, t+\tau) R\left(\mathbf{x}-\mathbf{x}^{\prime}\right) \tilde{s}\left(\mathbf{x}^{\prime}, t+\tau\right)\right) .
\end{aligned}
$$

Here we have defined

$$
V(s)=D \nabla^{2} s+\frac{D g}{2}(\nabla s)^{2}
$$

and with $R^{-1}(\mathbf{x})$ denote the reciprocal kernel of $R$, i.e., $\int d^{d} x^{\prime \prime} R\left(\mathbf{x}-\mathbf{x}^{\prime \prime}\right) R^{-1}\left(\mathbf{x}^{\prime \prime}-\mathbf{x}^{\prime}\right)=\delta\left(\mathbf{x}-\mathbf{x}^{\prime}\right)$. We have also introduced the purely imaginary response field $\tilde{s}(\mathbf{x}, t)$ which corresponds to the adjungated operator in the operator formulation of statistical dynamics by Martin, Siggia, and Rose [29. Note that the last expression in (2.8) is properly normalized,

$$
\int \mathcal{D}[s(t+\tau)] \mathcal{P}[s(t+\tau) \mid s(t)]=1,
$$

if one includes a purely numerical constant, independent of any parameter of the theory, in the functional measure $\mathcal{D}[\tilde{s}(t), s(t)]$. The joint probability distribution can be expressed in terms of a Markoff chain

$$
\prod_{k=1}^{K} \mathcal{P}[s(t+k \tau) \mid s(t+(k-1) \tau)] .
$$

Upon taking the limit $\tau \rightarrow 0, K \rightarrow \infty$ with $\tau K=T=$ const., we finally arrive at the path probability distribution

$$
\begin{aligned}
\mathcal{P} & {\left[s, 0<t \leq T \mid s(t=0)=s_{0}\right] } \\
& =\left.\int \mathcal{D}[\tilde{s}] \exp \left(-\mathcal{J}_{0}^{T}[\tilde{s}, s]\right)\right|_{s(t=0)=s_{0}}
\end{aligned}
$$

for the field configurations $\{s(\mathbf{x}, t)\}$ in the time interval $0<t \leq T$, starting from a given initial configuration $\left\{s(\mathbf{x}, 0)=s_{0}(\mathbf{x})\right\}$. The dynamic functional 30,31,32,33 is given by

$$
\begin{aligned}
\mathcal{J}_{0}^{T}[\tilde{s}, s]= & \int_{0}^{T} d t \int d^{d} x\left\{\tilde { s } ( \mathbf { x } , t ) \left[\dot{s}(\mathbf{x}, t)-D \nabla^{2} s(\mathbf{x}, t)\right.\right. \\
& \left.-\frac{D g}{2}(\nabla s(\mathbf{x}, t))^{2}\right] \\
& \left.-\int d^{d} x^{\prime} \tilde{s}(\mathbf{x}, t) R\left(\mathbf{x}-\mathbf{x}^{\prime}\right) \tilde{s}\left(\mathbf{x}^{\prime}, t\right)\right\} .
\end{aligned}
$$

Expectation values can now be calculated by means of path integrals with the exponential weight $\exp (-\mathcal{J}[\tilde{s}, s])$. Perturbational expansions are then to be generated in usual diagrammatical terms, and form the basis for a renormalized dynamical field theory [34,35.

But only in $d=1$ and without correlated noise can the dynamic functional be written in the detailed-balance form

$$
\mathcal{J}_{0}^{T}[\tilde{s}, s]=\int_{0}^{T} d t \int d x\left\{\tilde{s}\left[\dot{s}+\mathcal{M} \frac{\delta \mathcal{H}}{\delta s}\right]-\tilde{s} \mathcal{M} \tilde{s}\right\},
$$

where the integral-kernel $\mathcal{M}$ is defined by

$$
\begin{aligned}
\mathcal{M}(x, y)= & D\left[\delta(x-y)+\frac{g}{3}\left(\theta(x-y) \frac{\partial s(x)}{\partial x}\right.\right. \\
& \left.\left.-\theta(y-x) \frac{\partial s(y)}{\partial y}\right)\right],
\end{aligned}
$$

with the hamiltonian $\mathcal{H}$ given by Eq. (2.7). Obviously, $\mathcal{J}$ now obeys the time inversion symmetry [32, 33.

$$
\begin{aligned}
s(x, t) \rightarrow & s(x,-t), \\
\tilde{s}(x, t) \rightarrow & -\tilde{s}(x,-t)+\left.\frac{\delta \mathcal{H}}{\delta s}\right|_{x,-t} \\
& =-\tilde{s}(x,-t)-\nabla^{2} s(x, t), \\
\mathcal{J}_{0}^{T}+\left.\mathcal{H}\right|_{t=0} \rightarrow & \mathcal{J}_{-T}^{0}+\left.\mathcal{H}\right|_{t=-T} .
\end{aligned}
$$

As the transition probability distribution is given by the path integral

$$
\begin{aligned}
\mathcal{P}\left[s_{T}, T \mid s_{0}, 0\right]= & \int \mathcal{D}[\tilde{s}, s] \prod_{x} \delta\left(s(x, T)=s_{T}(x)\right) \\
& \times\left.\exp \left(-\mathcal{J}_{0}^{T}[\tilde{s}, s]\right)\right|_{s(x, 0)=s_{0}(x)},(2 .
\end{aligned}
$$


one derives directly from (2.16) the detailed balance condition

$$
\mathcal{P}\left[s_{T}, T \mid s_{0}, 0\right] \mathcal{P}_{s t}\left[s_{0}\right]=\mathcal{P}\left[s_{0}, 0 \mid s_{T},-T\right] \mathcal{P}_{s t}\left[s_{T}\right],
$$

which implies that $\mathcal{P}_{s t}[s]$ in (2.7) is indeed the stationary probability distribution for $d=1$. In Appendix A, we present an alternative derivation of this result on the basis of the associated Fokker-Planck equation.

In the following we shall not be interested in the initialtime properties of the stochastic process. Thus we extend the time interval in the expression for the dynamic functional from minus to plus infinity, which then reduces to

$$
\begin{aligned}
& \mathcal{J}=\int_{x} \int_{t} \tilde{s}\left[\partial_{t} s-D \nabla^{2} s-\frac{D g}{2}(\nabla s)^{2}\right] \\
& -\int_{q} \int_{\omega} D \tilde{s}(-\mathbf{q},-\omega)\left(1+w q^{-2 \rho}\right) \tilde{s}(\mathbf{q}, \omega) .
\end{aligned}
$$

Here, we have employed the short-hand notation $\int_{q} \ldots=$ $\int \ldots d^{d} q /(2 \pi)^{d}$ and $\int_{\omega} \ldots=\int \ldots d \omega / 2 \pi$.

We finally note that the dynamic functional is invariant under Galilean transformations

$$
\begin{aligned}
& s(\mathbf{x}, t) \rightarrow s(\mathbf{x}+D g \mathbf{v} t, t)+\mathbf{v} \cdot \mathbf{x}, \\
& \tilde{s}(\mathbf{x}, t) \rightarrow \tilde{s}(\mathbf{x}+D g \mathbf{v} t, t) .
\end{aligned}
$$

Implications of this Galilean invariance, specifically on the renormalizability of the KPZ dynamic field theory, are discussed in Appendix B.

\subsection{Renormalization and scaling}

We proceed by introducing renormalized quantities and the associated renormalization constants. In the "standard" representation of the KPZ problem (2.1), there appears no field renormalization [36]. Furthermore, Galilean invariance of the noisy Burgers equation, or infinitesimal interface tilt invariance for the KPZ problem, implies that the non-linear coupling $\lambda$ does not renormalize either, which leaves only the two independent renormalization factors for the diffusion constant $D$ and the noise strength $\widetilde{D}$ to be determined. This remains true even for the situation with power-law correlated spatial noise, for which Galilean invariance of course still holds. Furthermore, the long-range noise contributions do enter the renormalization of the diffusion constant and the short-range noise amplitude (see Sec. 3); in fact, even if $\widetilde{D}$ were zero initially, such spatially local noise terms would be generated under renormalization. However, the amplitude of the spatially correlated noise $\widetilde{\Delta}$ itself is not renormalized, because nonanalytic terms of the form $\propto q^{-2 \rho}$ can never be produced in a perturbation expansion for the corresponding vertex function (evaluated at non-zero external wave vector $q$ ).

In our representation in section 2.1, on the other hand, we have absorbed the ratio $D / \widetilde{D}$ in the new fields $s$, which leads to a non-trivial field renormalization [37]. However, the diffusive character of the fluctuations, and the wavevector dependence of the non-linear vertices still implies the exact result (to all orders in perturbation theory) [36,38

$$
\Gamma_{\tilde{s} s}(\mathbf{q}=\mathbf{0}, \omega) \equiv i \omega,
$$

and we can therefore define renormalized fields according to

$$
\begin{aligned}
& s \rightarrow s_{0}=Z^{\frac{1}{2}} s, \\
& \tilde{s} \rightarrow \tilde{s}_{0}=Z^{-\frac{1}{2}} \tilde{s} .
\end{aligned}
$$

The above considerations still remain valid, and we merely need to introduce one more independent renormalization constant $Z_{D}$ via

$$
D \rightarrow D_{0}=Z Z_{D} D
$$

The non-renormalization of the three-point vertex and the non-analytic long-range noise amplitude then implies that we can introduce corresponding dimensionless renormalized parameters $u$ and $v$ via

$$
g^{2} \rightarrow g_{0}^{2}=Z^{-3} Z_{D}^{-2} u A_{d}^{-1} \mu^{2-d},
$$

and

$$
w g^{2} \rightarrow w_{0} g_{0}^{2}=\left(Z Z_{D}\right)^{-3} v B_{d}^{-1}(\rho) \mu^{2(1+\rho)-d} .
$$

Eqs. (2.24) and (2.25) define the two effective couplings associated with the combination of the non-linearity in the Langevin equation with the local and power-law noise correlations, respectively. For convenience, we shall choose the following geometric factors

$$
A_{d}:=\frac{\Gamma\left(2-\frac{d}{2}\right)}{2^{d-1} \pi^{\frac{d}{2}} d} \sin \left(\frac{\pi d}{4}\right),
$$

and

$$
B_{d}(\rho):=\frac{\Gamma\left(2+\rho-\frac{d}{2}\right) \Gamma\left(\frac{d}{2}-\rho\right)}{2^{d-1} \pi^{\frac{d}{2}} d \Gamma\left(\frac{d}{2}\right)} \sin \left(\frac{\pi(d-2 \rho)}{4}\right),
$$

where $\Gamma(x)=\Gamma(1+x) / x$ denotes Euler's gamma function. The momentum scale $\mu$ represents an appropriately chosen renormalization point outside the infrared-singular region, e.g., $\mathbf{q}=\mathbf{0}$, and $\omega / 2 D=\mu^{2}$.

The asymptotic scaling behavior of the theory is governed by the properties in the vicinity of the renormalization-group fixed points $u_{*}$ and $v_{*}$, defined as the zeros of the RG beta functions

$$
\beta_{u}=\left.\mu \frac{\partial u}{\partial \mu}\right|_{0}, \quad \text { and } \quad \beta_{v}=\left.\mu \frac{\partial v}{\partial \mu}\right|_{0},
$$

which describe the dependence of the renormalized nonlinear couplings on the momentum scale $\mu$; the above derivatives are to be taken after re-expressing $u$ and $v$ in terms of their bare counterparts $g_{0}^{2}$ and $w_{0} g_{0}^{2}$. In addition, we define the Wilson functions

$$
\gamma=\left.\frac{\partial \ln Z}{\partial \ln \mu}\right|_{0}, \quad \gamma_{D}=\left.\frac{\partial \ln Z_{D}}{\partial \ln \mu}\right|_{0},
$$


yielding the anomalous dimensions for the field $s$ and the diffusion constant $D$, respectively, and

$$
\zeta=\left.\frac{\partial \ln D}{\partial \ln \mu}\right|_{0}=-\gamma-\gamma_{D} .
$$

Eqs. (2.24) and (2.25) then imply the following relations

$$
\begin{aligned}
& \beta_{u}=\left(d-2+3 \gamma+2 \gamma_{D}\right) u, \\
& \beta_{v}=\left(d-2-2 \rho+3 \gamma+3 \gamma_{D}\right) v .
\end{aligned}
$$

The fact that the bare correlation function $C_{0}(x, t)$ is of course independent of the chosen normalization point $\mu$, leads to the Gell-Mann-Low renormalization-group equation (see, e.g., Refs. 30,36] )

$$
\begin{gathered}
{\left[\mu \frac{\partial}{\partial \mu}+\gamma_{D} D \frac{\partial}{\partial D}+\beta_{u} \frac{\partial}{\partial u}+\beta_{v} \frac{\partial}{\partial v}+\gamma\right]} \\
\times C(x, t ; u, v ; D, \mu)=0
\end{gathered}
$$

for the renormalized correlation function $C(x, t)$,

$$
\left\langle s(x, t) s\left(x^{\prime}, t^{\prime}\right)\right\rangle=C\left(x-x^{\prime}, t-t^{\prime}\right) .
$$

The standard solution of the partial differential equation (2.33) proceeds via the method of characteristics, whereupon we parametrize $\mu(\ell)=\mu \ell$. For the correlation function, this leads to

$$
\begin{aligned}
C(x, t ; u, v ; D, \mu)=\exp \left[\int_{1}^{\ell} \gamma\left(\ell^{\prime}\right) \frac{d \ell^{\prime}}{\ell^{\prime}}\right] \\
\quad \times C(x, t ; u(\ell), v(\ell) ; D(\ell), \mu \ell) \\
=(\mu \ell)^{d-2} \exp \left[\int_{1}^{\ell} \gamma\left(\ell^{\prime}\right) \frac{d \ell^{\prime}}{\ell^{\prime}}\right] \\
\quad \times \hat{C}\left(\mu \ell x,(\mu \ell)^{2} D(\ell) t ; u(\ell), v(\ell)\right) .
\end{aligned}
$$

In the last step, we have explicitly extracted the scaling dimension of the fields $\left([s(\mathbf{x}, t)]=\mu^{(d-2) / 2}\right)$, and thus rendered the quantity $\hat{C}$ dimensionless. The "running couplings" are furthermore given by the solution of the flow equations

$$
\begin{aligned}
& \ell \frac{d u(\ell)}{d \ell}=\beta_{u}(u(\ell), v(\ell)), \\
& \ell \frac{d v(\ell)}{d \ell}=\beta_{v}(u(\ell), v(\ell)),
\end{aligned}
$$

and

$$
\ell \frac{d D(\ell)}{d \ell}=D(\ell) \zeta(u(\ell), v(\ell)) .
$$

In the vicinity of a renormalization-group fixed point, Eq. (2.36) reduces to

$$
C(x, t) \propto \ell^{d-2+\gamma^{*}} \hat{C}\left(\mu \ell x, \mu^{2} D \ell^{2+\zeta^{*}} t ; u_{*}, v_{*}\right),
$$

with $\gamma^{*}=\gamma\left(u_{*}, v_{*}\right)$ and $\zeta^{*}=\zeta\left(u_{*}, v_{*}\right)$. Upon employing the matching condition $\mu \ell x=1$, one finally obtains the identification of the scaling exponents with the above anomalous dimensions $\gamma^{*}$ and $\zeta^{*}$ at a stable RG fixed point.

Using the conventional notation for dynamic critical phenomena, we write the two-point correlation function in the scaling form

$$
C(x, t)=\frac{1}{x^{d-2+\eta}} \hat{C}\left(t / x^{z}\right) .
$$

Here, $\eta$ and $z$ are the Wilson-Fisher and dynamic scaling exponents, respectively, and $\hat{C}(y)$ is a scaling function with the asymptotic limits $\hat{C}(y) \rightarrow$ const. for $y \rightarrow 0$, and $\hat{C}(y) \rightarrow y^{(2-d-\eta) / z}$ for $y \rightarrow \infty$. In Fourier space, Eq. (2.40) becomes

$$
C(q, \omega)=\frac{1}{q^{z+2-\eta}} \hat{C}\left(\omega / q^{z}\right) .
$$

In terms of the anomalous dimensions $\gamma^{*}$ and $\zeta^{*}$, according to Eq. (2.39) these two independent exponents read

$$
\eta=\gamma^{*}, \quad \text { and } z=2+\zeta^{*} .
$$

In the context of non-equilibrium growth and interface scaling phenomena, Eq. (2.40) is usually expressed in terms of the roughness exponent $\chi$ instead of $\eta$,

$$
C(x, t)=x^{2 \chi} \hat{C}\left(t / x^{z}\right) .
$$

Thus we identify

$$
\chi=\frac{2-d-\eta}{2}=\frac{2-d-\gamma^{*}}{2} .
$$

For the Gaussian (Edwards-Wilkinson) model with $g=0$, which corresponds to a simple noisy diffusion equation, one immediately obtains $\eta=0, z=2$, and $\chi=1-\frac{d}{2}$.

We finally note that any finite, non-zero fixed point $0<u_{*}<\infty$ implies $d-2+\gamma^{*}-2 \zeta^{*}=0$, see (2.31), and therefore the non-trivial scaling relation

$$
z+\chi=2 .
$$

In the same manner, Eq. (2.32) leads to the additional exact result

$$
z_{\mathrm{lr}}=\frac{4+d-2 \rho}{3},
$$

provided $0<v_{*}<\infty$. In situations where Eq. (2.45) holds as well, this already fixes the scaling exponents of the KPZ problem with stable long-range correlated noise to

$$
\eta_{\operatorname{lr}}=\frac{2-d-4 \rho}{3}, \quad \chi_{\operatorname{lr}}=\frac{2-d+2 \rho}{3} .
$$

Furthermore, if $\beta_{v}$ is strictly positive, then $v \rightarrow 0$ under scale transformations, and the short-range fixed point is stable against the long-range noise correlations. In terms of the short-range dynamic exponent $z_{\mathrm{sr}}$, Eq. (2.32) thus implies the exact stability condition

$$
z_{\mathrm{sr}}<z_{\mathrm{lr}}=\frac{4+d-2 \rho}{3}
$$




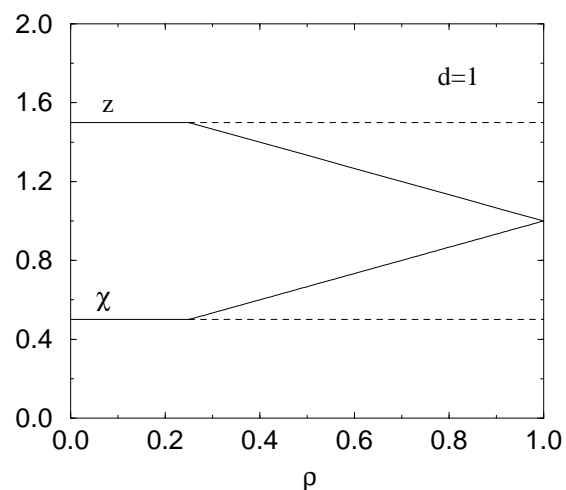

Fig. 2.1. Exact roughness and dynamic exponent as a function of the exponent $\rho$ of the noise correlations in the $(1+1)-$ dimensional model. Above a critical value $\rho_{c}=\frac{1}{4}$ the longrange fixed point becomes stable.

for the KPZ fixed point with local noise.

In one dimension, and for purely short-range noise, i.e., $w=0$, we know that the stationary distribution is given by (2.7). Thus we immediately see that the statics is characterized by Gaussian exponents, and hence

$$
d=1: \quad \eta_{\mathrm{sr}}=0, \quad \chi_{\mathrm{sr}}=\frac{1}{2}, \quad z_{\mathrm{sr}}=\frac{3}{2},
$$

where we have used the scaling relations (2.44) and (2.45), which is valid in the non-linear regime. Eq. (2.48) then yields the stability condition for the short-range fixed point to all orders of perturbation theory, namely

$$
\rho<\frac{1}{4} \Longrightarrow \text { short }- \text { range fixed point stable }
$$

The task of an explicit renormalization group analysis is therefore to establish the fixed-point structure of the field theory (2.19). It turns out that to this end one has to distinguish the cases $d<2(1+\rho)$ and $d \geq 2(1+\rho)$. For, as can be already inferred from the naive scaling dimension of the long-range coupling $v$, see Eqs. (2.25) and (2.32),

$$
d_{c}=2(1+\rho)
$$

defines a lower critical dimension for the roughening transition in the KPZ problem with long-range spatially correlated noise. In the following two sections, we shall discuss the distinct scaling regimes below and above the lower critical dimension (2.51) separately.

\section{Scaling behavior below the critical dimension}

\subsection{Non-minimal renormalization at fixed dimension}

We start by evaluating the renormalization constants for fixed dimension $d$ and correlation exponent $\rho$. The two independent $Z$ factors $Z$ and $Z_{D}$ can be determined by evaluating the two-point vertex functions $\partial_{q^{2}} \Gamma_{\tilde{s} s}(\mathbf{q}, \omega)$ and $\Gamma_{\tilde{s} \tilde{s}}(\mathbf{q}, \omega)$ at the normalization point $\mathbf{q}=\mathbf{0}, \omega / 2 D=$ $\mu^{2}$. The corresponding one-loop diagrams are identical to those for the conventional KPZ problem with short-range noise, see e.g. Ref. [36], and yield to leading order in the external momentum $\mathbf{q}$

$$
\begin{gathered}
\Gamma_{\tilde{s} s}(q, \omega)=i \omega+D q^{2}\left[1-\frac{d-2}{4 d} g^{2} \int_{p} \frac{1}{p^{2}+i \omega / 2 D}\right. \\
\left.-\frac{d-2(1+\rho)}{4 d} w g^{2} \int_{p} \frac{p^{-2 \rho}}{p^{2}+i \omega / 2 D}\right]
\end{gathered}
$$

and

$$
\begin{gathered}
\Gamma_{\tilde{s} \tilde{s}}(q, \omega)=-2 D\left[1+w q^{-2 \rho}+\frac{g^{2}}{4} \Re \int_{p} \frac{1}{p^{2}+i \omega / 2 D}\right. \\
\left.+\frac{w g^{2}}{2} \Re \int_{p} \frac{p^{-2 \rho}}{p^{2}+i \omega / 2 D}+\frac{w^{2} g^{2}}{4} \Re \int_{p} \frac{p^{-4 \rho}}{p^{2}+i \omega / 2 D}\right] .
\end{gathered}
$$

At the normalization point, and in dimensional regularization, the real parts of the above momentum integrals become

$$
\begin{gathered}
\Re \int_{p} \frac{1}{p^{2 \sigma}\left(p^{2}+i \mu^{2}\right)}=-\frac{\Gamma\left(2+\sigma-\frac{d}{2}\right) \Gamma\left(\frac{d}{2}-\sigma\right)}{2^{d-1} \pi^{\frac{d}{2}} \Gamma\left(\frac{d}{2}\right)} \\
\times \frac{\mu^{d-2(1+\sigma)}}{d-2(1+\sigma)} \sin \left[\frac{\pi}{4}(d-2 \sigma)\right] .
\end{gathered}
$$

Eq. (3.1) then leads to

$$
Z Z_{D}=1-\frac{1}{4} A_{d} g_{0}^{2} \mu^{d-2}\left(1+w_{0} \frac{B_{d}(\rho)}{A_{d}} \mu^{-2 \rho}\right)
$$

with the $d$-dependent coefficients $A_{d}$ and $B_{d}(\rho)$ from Eqs. (2.26) and (2.27). The vertex function (3.2) explicitly shows that the non-analytic contribution $\sim w_{0} q^{-2 \rho}$ is not renormalized by fluctuations. From Eq. (3.2) we find

$$
\begin{aligned}
Z_{D}=1 & +\frac{d A_{d}}{4(d-2)} g_{0}^{2} \mu^{d-2} \\
& +\frac{d B_{d}(\rho)}{2[d-2(1+\rho)]} w_{0} g_{0}^{2} \mu^{d-2(1+\rho)} \\
& +\frac{d B_{d}(2 \rho)}{4[d-2(1+2 \rho)]} w_{0}^{2} g_{0}^{2} \mu^{d-2(1+2 \rho)},
\end{aligned}
$$

and thus

$$
\begin{aligned}
Z=1 & -\frac{d-1}{2(d-2)} A_{d} g_{0}^{2} \mu^{d-2} \\
& -\frac{3 d-2(1+\rho)}{4[d-2(1+\rho)]} B_{d}(\rho) w_{0} g_{0}^{2} \mu^{d-2(1+\rho)} \\
& -\frac{d B_{d}(2 \rho)}{4[d-2(1+2 \rho)]} w_{0}^{2} g_{0}^{2} \mu^{d-2(1+2 \rho)} .
\end{aligned}
$$

Hence, there appear single poles in dimensional regularization at $d=2, d=2(1+\rho)$, and $d=2(1+2 \rho)$, above which 
the corresponding integrals become ultraviolet-divergent. Notice, however, that the product $Z Z_{D}$ in Eq. (3.4) is nonsingular. In a minimal renormalization scheme, one would therefore set $Z Z_{D}=1$ to one-loop order. While this is perfectly legitimate in the framework of an $\varepsilon$-expansion above the (lower) critical dimension, see Sec. \#, this minimal subtraction procedure would be inappropriate for $d<d_{c}$. This becomes already obvious in the short-range noise case $\left(w_{0}=0\right)$, where one would require that a fixed-dimension approach should recover the exact result $\eta_{\mathrm{sr}}=0$ in one dimension. Yet, this is clearly impossible with a non-trivial renormalization constant $Z=Z_{D}^{-1}=$ $1-d A_{d} g_{0}^{2} \mu^{d-2} / 4(d-2)$. On the other hand, the term $\sim g_{0}^{2}$ vanishes at $d=1$ in the "full" Z factor (3.6), as desired. In order to correctly incorporate the one-dimensional case into the theory, one has to apply a non-minimal renormalization scheme, and must retain not just the residues of the ultraviolet poles, but the full dimension dependence of the loop integrals 36.38]. For the KPZ problem with local noise correlator, an explicit two-loop analysis on this basis was performed in Ref. [36], which furthermore showed that the fixed-point value of the renormalized coupling $u$ diverges at the critical dimension $d_{c}=2$. In light of additional arguments presented in Ref. 39 that such a singularity is prone to emerge in any perturbational approach to the KPZ problem for $d \uparrow d_{c}$, this divergence clearly renders any attempt of an $\varepsilon$-expansion below $d_{c}$ obsolete.

Differentiation with respect to the normalization scale $\mu$, and expressing the result in terms of the renormalized quantities, yields to first order in the couplings $u$ and $v$,

$$
\zeta=\frac{d-2}{4} u+\frac{d-2(1+\rho)}{4} v,
$$

and

$$
\gamma=-\frac{d-1}{2} u-\frac{3 d-2(1+\rho)}{4} v-\frac{d}{4} h_{d}(\rho) \frac{v^{2}}{u} .
$$

Here we have defined the function

$$
\begin{aligned}
h_{d}(\rho)= & \frac{B_{d}(2 \rho) A_{d}}{B_{d}^{2}(\rho)} \\
= & \frac{B\left(2-\frac{d}{2}, \frac{d}{2}\right) B\left(2+2 \rho-\frac{d}{2}, \frac{d}{2}-2 \rho\right)}{\left[B\left(2+\rho-\frac{d}{2}, \frac{d}{2}-\rho\right)\right]^{2}} \\
& \times \frac{\sin \left[\frac{\pi}{4}(d-4 \rho)\right] \sin \left(\frac{\pi}{4} d\right)}{\sin ^{2}\left[\frac{\pi}{4}(d-2 \rho)\right]},
\end{aligned}
$$

using Euler's beta function $B(x, y)=\Gamma(x) \Gamma(y) / \Gamma(x+y)$. Combining the above results finally yields the $\mathrm{RG}$ beta functions

$$
\begin{aligned}
\beta_{u}(u, v)= & (d-2) u-\frac{2 d-3}{2} u^{2} \\
- & \frac{5 d-6(1+\rho)}{4} u v-\frac{d}{4} h_{d}(\rho) v^{2}, \\
\beta_{v}(u, v)= & (d-2-2 \rho) v-\frac{3}{4}(d-2) u v \\
& -\frac{3}{4}(d-2-2 \rho) v^{2} .
\end{aligned}
$$

\subsection{RG fixed points and scaling in one dimension}

The above one-loop beta functions (3.10) and (3.11) and ensuing flow equations (2.37) were essentially derived in Ref. [6] already, with the slight modification that there the function $h_{d}(\rho)$ was replaced with $h_{d}(0)=1$. This minor change does not drastically alter the scaling behavior as function of $\rho$ and $d$, however, and a discussion of the different regimes, albeit based on a one-loop approximation only, can be found in Ref. [6]. Here, we thus restrict ourselves to the analysis of the sole non-trivial physical realization below the critical dimension, namely $d=1$. We then have to distinguish between two different situations:

(i) $0 \leq \rho<\frac{1}{4}$ : In this case, apart from the Gaussian (Edwards-Wilkinson) fixed point $u_{*}=0, v_{*}=0$, there exists only the short-range KPZ fixed point

$$
u_{*}=2, \quad v_{*}=0,
$$

which furthermore is stable according to the exact result (2.50). The corresponding scaling exponents are those of (2.49). A numerical solution of the flow equations (2.37) for $\rho=0.2$ is shown in Fig. 3.1.

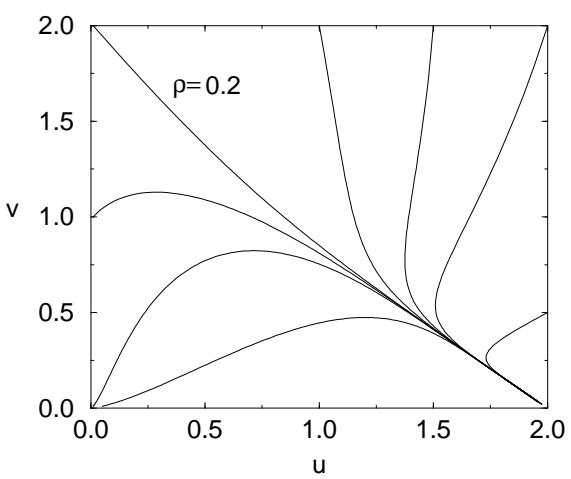

Fig. 3.1. Numerical solution of the RG flow equations (flow diagram) for the one-dimensional KPZ equation with spatially long-range correlated noise characterized by an exponent $\rho=$ 0.2 : the short-range fixed point $\left(u_{*}, v_{*}\right)=(2,0)$ is stable.

(ii) $\frac{1}{4} \leq \rho<1$ : In this regime, the short-range fixed point (3.12) is unstable. There exists, however, also a nontrivial zero of the beta functions (3.10) and (3.11), which leads to a quadratic equation with the solution

$$
\begin{aligned}
u_{*} & =\frac{4}{3} \frac{1+2 \rho}{h_{1}(\rho)-\left(1-4 \rho^{2}\right)}\left[h_{1}(\rho)-\left(1-\rho-6 \rho^{2}\right)\right. \\
& \left.+\sqrt{\left(1-\rho-6 \rho^{2}\right)^{2}-\left(1-2 \rho-8 \rho^{2}\right) h_{1}(\rho)}\right],(3 .
\end{aligned}
$$

and

$$
v_{*}=\frac{4}{3}-\frac{u_{*}}{1+2 \rho} .
$$

The non-trivial long-range fixed point is stable, as can be seen in the flow diagram for the case $\rho=0.4$, which is 
depicted in Fig. 3.2. From (2.46) and (2.47) we therefore infer the values for the long-range noise scaling exponents in one dimension,

$$
\eta_{\mathrm{lr}}=\frac{1-4 \rho}{3}<0, \quad \chi_{\mathrm{lr}}=\frac{1+2 \rho}{3}
$$

and

$$
z_{\mathrm{lr}}=\frac{5-2 \rho}{3}
$$

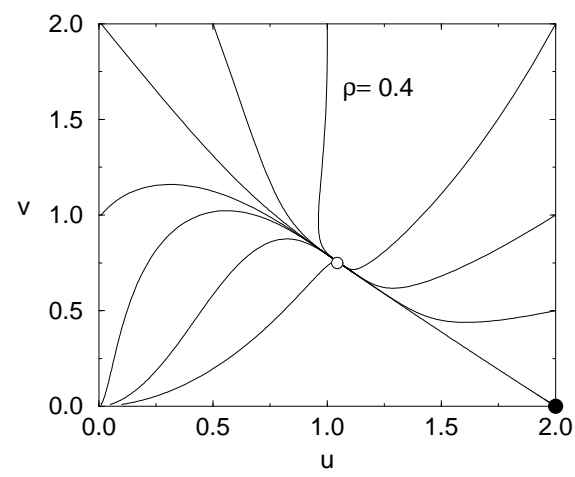

Fig. 3.2. Numerical solution of the RG flow equations (flow diagram) for the one-dimensional KPZ equation with spatially long-range correlated noise characterized by an exponent $\rho=$ 0.4: The short-range fixed point becomes unstable, and the stable long-range fixed point $\left(u_{*}, v_{*}\right)$ governs the asymptotic scaling behavior.

(iii) $\rho>1$ : For $\rho>1$ the above exact results for the dynamic exponent become invalid, since $z$ becomes smaller than 1, which would imply motion faster than ballistic transport. Surprisingly, however, Hayot and Jayaprakash 22,23 find agreement with the exact result for the roughness exponent $\chi$ even for $\rho \geq 1$. This interesting observation still lacks a deeper theoretical understanding.

Thus, despite the uncontrolled non-minimal renormalization prescription which does not allow for the identification of a small expansion parameter in the perturbation series, we could obtain the exact results (2.49) and (3.15) for the scaling exponents at the short-range and longrange fixed points, respectively, as well as for the stability criterion (2.50). In the short-range case, the "hidden" fluctuation-dissipation theorem in one dimension, and the scaling relation (2.45) are sufficient to fix the scaling exponents. For power-law correlated noise, which cannot be renormalized perturbatively, the additional condition of a non-zero, finite fixed point $v_{*}$ determined $z$, and via Eq. 2.45 $\chi$ as well. This remarkable fact is also intimately related to the mapping of the noisy Burgers equation to a driven diffusive system in one dimension. As opposed to the KPZ problem, driven diffusive systems do permit a systematic $\varepsilon$ expansion below $d_{c}=2$, and are therefore subject to a controlled perturbation expansion 40]. A direct analysis of driven diffusive systems with long-range correlated spatial noise in fact yields the identical stability condition (2.50) for the short-range fixed point, and confirms the result (3.15) 41.

\section{The roughening transition and scaling above the critical dimension}

Above the critical dimension $d_{c}=2(1+\rho)$, a systematic perturbational expansion in terms of $d-d_{c}$ is possible within a minimal renormalization scheme. As was demonstrated to two-loop order in Ref. [36], the shortrange noise KPZ equation has a non-trivial unstable fixed point for $d \geq d_{c}=2$, which vanishes as $d \rightarrow 2$ (see also Ref. 42]). It is therefore possible to design a controlled $(2+\varepsilon)$-expansion with a well-defined small parameter, as opposed to the situation below the critical dimension. The unstable fixed point describes a non-equilibrium roughening transition, separating a smooth phase with EdwardsWilkinson scaling exponents from a strong-coupling rough phase, which remains inaccessible through perturbational means 39. The $(2+\varepsilon)$-expansion itself works in very close analogy with the standard field-theoretic treatment of the non-linear sigma model, where the unstable fixed point corresponds to the critical temperature (see Appendix B). Upon employing the directed-polymer representation of the KPZ problem, it was subsequently demonstrated that the one-loop beta function obtained within such an $\varepsilon$ expansion is actually exact to all orders in perturbation theory $39,43,44$.

The KPZ problem with power-law correlated noise can be treated in much the same manner. As in other problems where non-analytic long-range expressions are present in the bare vertices, the expansion about the critical dimension has to be supplemented with an expansion with respect to the exponent $\rho$ 45,46,47,48. Correspondingly, we may construct a minimal renormalization scheme, where the residues of the $\varepsilon=d-2$ poles in the $Z$ factors are to be taken at $d=2$ and $\rho=0$. For our previous one-loop results, this immediately means that

$$
Z Z_{D}=1
$$

and therefore

$$
\zeta=0, \quad \text { and } \gamma=-\frac{(u+v)^{2}}{2 u}
$$

in minimal subtraction. Notice that the above result for $\zeta$ implies $z=2$ at any RG fixed point, see Eq. (2.42). Furthermore, Eqs. (2.31) and (2.32) yield the one-loop RG beta functions

$$
\begin{aligned}
& \beta_{u}(u, v)=\varepsilon u-\frac{1}{2}(u+v)^{2}, \\
& \beta_{v}(u, v)=(\varepsilon-2 \rho) v .
\end{aligned}
$$

The same expressions may of course be obtained by setting $d=2$ and $\rho=0$ in the quartic terms in (3.10) and (3.11).

Notice that by using an expansion with respect to $\rho$, we restrict ourselves to fixed points which are of the same 
type as the fixed points at $\rho=0$, i.e., fixed points describing the smooth phase and the roughening transition of the short-range problem. We cannot expect to learn anything about the strong-coupling regime this way. In general the fixed-point structure may radically change as a function of $\rho$. In fact, this must be the case for the KPZ equation, as may be inferred from considering the particular case $\rho=1$, which corresponds to the Burgers equation with non-conserved noise ("model B" in the notation of Forster et al. [15]). The latter model displays no non-equilibrium phase transition, and is known to have an upper critical dimension $d_{c}=4$. A straightforward renormalization-group analysis of the Burgers equation with non-conserved noise 15. 14 tells us that the dynamic exponent is exactly given by $z=(2+d) / 3$, and that there is a stable fixed point $v_{*}$ to order $4-d$. From our general analysis in section 2 (which is valid to any order in perturbation theory), we also know that this exponent is the analytic continuation of the strong-coupling (long-range noise) fixed point. By using an expansion with respect to $\rho$, this fixed point will obviously not be accessible. Hence, there must be some borderline in the $(d, \rho)$-plane where a non-analytic change in the fixed-point structure takes place. We shall return to this issue in our final section 6. For now, we restrict ourselves mainly to a discussion of the roughening transition above the critical dimension $d_{c}=2(1+\rho)$.

\subsection{Cole-Hopf transformation and minimal renormalization above the critical dimension}

We will show in this section that, similar to the shortrange case [39], the above one-loop RG beta functions actually hold to all orders in perturbation theory. The simplest way to see this is via a stochastic Cole-Hopf transformation 43. (see also Refs. 49,44), which maps the KPZ equation onto the problem of directed polymers in a random environment [50,51,52,53]. Hence we define new fields $n(\mathbf{x}, t)$ via

$$
n(\mathbf{x}, t)=\frac{2}{g} \exp \left[\frac{g}{2} s(\mathbf{x}, t)\right] .
$$

In the discretized form of the dynamic functional resulting from (2.8), this highly non-linear transformation is to be supplemented with

$$
\tilde{n}(\mathbf{x}, t+\tau)=\tilde{s}(\mathbf{x}, t+\tau) \exp \left[-\frac{g}{2} s(\mathbf{x}, t)\right],
$$

which renders the Cole-Hopf transformation from the fields $(s, \tilde{s})$ to $(n, \tilde{n})$ pseudo-canonical, i.e., with Jacobian

$$
\frac{\partial(n, \tilde{n})}{\partial(s, \tilde{s})}=1, \quad \text { and } \quad \tilde{s} \frac{\partial s}{\partial t}=\tilde{n} \frac{\partial n}{\partial t},
$$

as long as one neglects the time increment $\tau$, and considers the fields as analytic functions. However, we need to be slightly more careful here since it is known [33] that the time derivatives of the fields as well as the response fields are actually nowhere continuous. Thus, by means of such a non-linear transformation, one has to take recourse to the discretized form of the functional, or alternatively use different methods like Fokker-Planck equations or Langevin equations in a well defined form 54. Then one finds that the apparently trivial terms in Eqs. (4.7) produce a nonstraightforward contribution $-D g R(0) \tilde{n} n / 2$ to the integrand of the dynamic functional 2.13), which is proportional to the noise. Furthermore,

$$
\nabla \tilde{n} \nabla n=\nabla \tilde{s} \nabla s-g \tilde{s}(\nabla s)^{2} / 2,
$$

which eliminates the non-linear term in the effective equation of motion, leaving only the noise vertex. The dynamic functional in terms of the fields $n$ and $\tilde{n}$ thus becomes

$$
\begin{gathered}
\mathcal{J}=\int d t\left\{\int _ { x } \tilde { n } ( \mathbf { x } , t ) \left[\dot{n}(\mathbf{x}, t)-D \nabla^{2} n(\mathbf{x}, t)\right.\right. \\
\left.-\frac{D g}{2} R(0) n(\mathbf{x}, t)\right] \\
\left.-\frac{D g^{2}}{4} \int_{x} \int_{x^{\prime}} \tilde{n}(\mathbf{x}, t) n(\mathbf{x}, t) R\left(\mathbf{x}-\mathbf{x}^{\prime}\right) \tilde{n}\left(\mathbf{x}^{\prime}, t\right) n\left(\mathbf{x}^{\prime}, t\right)\right\} .
\end{gathered}
$$

Now it becomes obvious that the seemingly problematic term $\propto R(0)$ can be eliminated by introducing an additional term in the stochastic Cole-Hopf transformation: one merely has to replace $s(\mathbf{x}, t) \rightarrow s(\mathbf{x}, t)+D R(0) t$ in the exponentials of Eqs. (4.5) and (4.6). This justifies our dropping this term in the following, whereupon we write the dynamic functional in terms of the fields $n$ and $\tilde{n}$ in the form

$$
\begin{aligned}
\mathcal{J}= & \int d^{d} x \int d t\left[\tilde{n}\left(\partial_{t} n-D \nabla^{2} n\right)\right. \\
& \left.-\frac{D g^{2}}{4}\left([\tilde{n} n]^{2}+w\left[\nabla^{-\rho}(\tilde{n} n)\right]^{2}\right)\right] .
\end{aligned}
$$

When we introduce renormalized quantities according to Eqs. (2.22)-(2.25), and furthermore demand that the relations (4.5) and (4.6) hold for the renormalized fields as well, we find

$$
\tilde{n} n \rightarrow Z Z_{D} \tilde{n} n,
$$

and the renormalized action reads

$$
\begin{aligned}
& \mathcal{J}=\int d^{d} x \int d t\left[Z Z_{D} \tilde{n}\left(\partial_{t} n-Z Z_{D} D \nabla^{2} n\right)\right. \\
& \left.-\frac{D \mu^{2-d}}{4}\left(Z_{D} u A_{d}^{-1}(\tilde{n} n)^{2}+v B_{d}^{-1} \mu^{2 \rho}\left[\nabla^{-\rho}(\tilde{n} n)\right]^{2}\right)\right] .
\end{aligned}
$$

However, in the field theory $(4.10)$ with the bare diffusion propagator $\left(-i \omega+D q^{2}\right)^{-1}$ and the four-point noise vertex depicted in Fig. 4.1, there exist no loop diagrams contributing to the renormalization of the propagator. Therefore, in a minimal renormalization scheme, Eq. (4.1), and hence Eq. (4.4) hold to all orders in perturbation theory. This leaves us with the determination of $Z_{D}$ from 


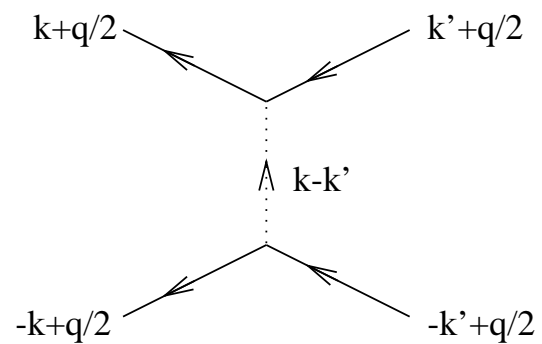

Fig. 4.1. Four-point vertex for the KPZ problem with spatially long-range correlated noise in the Cole-Hopf representation.
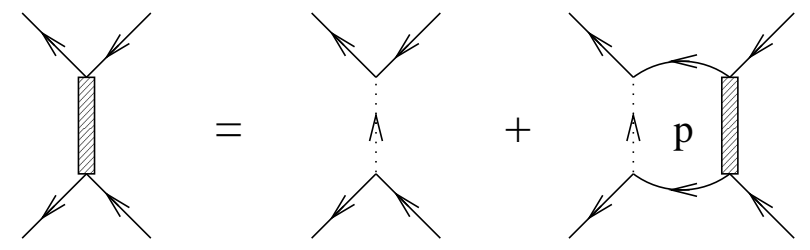

Fig. 4.2. Diagrammatic representation of the Bethe-Salpeter equation for the four-point noise vertex.

the renormalization of the noise vertex. As a consequence of the absence of any propagator renormalizations, this is readily achieved via a summation of the entire perturbation series by means of a Bethe-Salpeter equation, see Fig. 4.2. This is actually in complete analogy with the field-theoretic analysis of diffusion-limited pair annihilation reactions, see Ref. [25,26,27]. Upon defining

$$
\begin{gathered}
\Gamma_{22}\left(\mathbf{k}, \mathbf{k}^{\prime} ; \frac{i \omega}{2 D}+\frac{q^{2}}{4}\right)=-\Gamma_{\tilde{n} \tilde{n} n n}\left(-\frac{\mathbf{q}}{2}-\mathbf{k},-\frac{\omega}{2}\right. \\
\left.-\frac{\mathbf{q}}{2}+\mathbf{k},-\frac{\omega}{2} ; \frac{\mathbf{q}}{2}+\mathbf{k}^{\prime}, \frac{\omega}{2} ; \frac{\mathbf{q}}{2}-\mathbf{k}^{\prime}, \frac{\omega}{2}\right)
\end{gathered}
$$

the corresponding analytic expression, taken at the normalization point $i \omega / 2 D+q^{2} / 4=\mu^{2}$, reads

$$
\begin{aligned}
\Gamma_{22}\left(\mathbf{k}, \mathbf{k}^{\prime} ; \mu^{2}\right)=D g^{2} R\left(\mathbf{k}-\mathbf{k}^{\prime}\right) \\
+\frac{g^{2}}{4} \int_{p} \frac{R(\mathbf{k}-\mathbf{p})}{\mu^{2}+p^{2}} \Gamma_{22}\left(\mathbf{p}, \mathbf{k}^{\prime} ; \mu^{2}\right),
\end{aligned}
$$

where $R(\mathbf{k})=1+w k^{-2 \rho}$.

In order to determine the renormalization factors from the Bethe-Salpeter equation, it suffices to consider it at $\mathbf{k}^{\prime}=\mathbf{0}$ and to leading order in the external momentum $\mathbf{k}$. Then the Bethe-Salpeter equation reduces to

$$
\begin{aligned}
& \Gamma_{22}\left(\mathbf{k}, \mathbf{0} ; \mu^{2}\right)=D g^{2}\left(1+w k^{-2 \rho}\right) \\
& \quad+\frac{g^{2}}{4} \int_{p} \frac{1+w p^{-2 \rho}}{\mu^{2}+p^{2}} \Gamma_{22}\left(\mathbf{p}, \mathbf{0} ; \mu^{2}\right) .
\end{aligned}
$$

Upon introducing $Z$ factors through Eqs. (2.23), (2.24), and (2.25), taking into account the identity (4.1) and requiring that to leading order in the momentum the renormalized vertex function assumes the form

$$
\Gamma_{22}\left(\mathbf{k}, \mathbf{0} ; \mu^{2}\right)=D \mu^{2-d}\left[u A_{d}^{-1}+v B_{d}^{-1}(\mu / k)^{2 \rho}\right],
$$

one finds

$$
\begin{gathered}
u A_{d}^{-1} \mu^{2-d}\left[1-\frac{g_{0}^{2}}{4} \int_{p} \frac{1+w_{0} p^{-2 \rho}}{p^{2}+\mu^{2}}\right] \\
=g_{0}^{2}\left[1+\frac{w_{0} g_{0}^{2}}{4} \int_{p} \frac{1+w_{0} p^{-2 \rho}}{p^{2 \rho}\left(p^{2}+\mu^{2}\right)}\right] .
\end{gathered}
$$

Evaluating the integrals with the aid of the formula (3.3), we thus arrive at the exact result

$$
\begin{aligned}
Z_{D} & =\left(1+\frac{d A_{d} g_{0}^{2} \mu^{d-2}}{4(d-2)}+\frac{d B_{d} w_{0} g_{0}^{2} \mu^{d-2(1+\rho)}}{4[d-2(1+\rho)]}\right) \\
& \times\left(1-\frac{d B_{d} w_{0} g_{0}^{2} \mu^{d-2(1+\rho)}}{4[d-2(1+\rho)]}\right. \\
- & \left.\frac{\Gamma\left(2+2 \rho-\frac{d}{2}\right) \Gamma\left(\frac{d}{2}-2 \rho\right)}{2^{d-1} \pi^{\frac{d}{2}} \Gamma\left(\frac{d}{2}\right)} \frac{w_{0}^{2} g_{0}^{2} \mu^{d-2(1+2 \rho)}}{4[d-2(1+2 \rho)]}\right)^{-1} .
\end{aligned}
$$

When this is expanded with respect to first order in the fluctuation contributions, the one-loop result (3.5) is recovered. In the minimal renormalization scheme, however, we have to set $d=2+\varepsilon$ and $\rho=0$. The somewhat cumbersome expression 4.18 then becomes nicer when $Z_{D}$ is re-expressed in terms of the renormalized couplings,

$$
Z_{D}=\frac{1+\frac{1}{2 \varepsilon} v+\frac{1}{2 \varepsilon} \frac{v^{2}}{u}}{1-\frac{1}{2 \varepsilon} u-\frac{1}{2 \varepsilon} v} .
$$

In a similar manner, differentiating Eq. (4.18) with respect to the normalization scale $\mu$, and expressing the result in terms of $u$ and $v$ yields precisely the RG beta functions (4.3) and (4.4), which are thus valid to all orders in minimally renormalized perturbation theory.

Alternatively, one may utilize the structure of the perturbation expansion, combined with the minimal renormalization prescription, to demonstrate the validity of the beta functions (4.3) and (4.4) to all orders in the perturbation series. In the following, we employ a double expansion in $\varepsilon$ and $\rho$, where it is assumed that both quantities are of equal order of magnitude, in combination with minimal renormalization. Consider the perturbational expansion of the Bethe-Salpeter equation (4.2) with respect to $g^{2} \propto u$ and $w$. A contribution proportional to $g^{2 n} w^{k}$ $(k \leq n)$ is accompanied by a momentum integral that diverges superficially as $\Lambda^{n \varepsilon-k \rho}$, where $\Lambda$ denotes an ultraviolet momentum cutoff. First, let $\varepsilon<0$. Then, as shown by Honkonen and Nalimov 48 in the analogous case of the Landau-Ginzburg-Wilson hamiltonian with long-range interactions, the contributions are effectively regularized by a combination of the dimensional and the analytic regularization schemes. Instead of the UV divergences, now pole singularities of the combinations $\delta=-n \varepsilon+$ $2 k \rho$ arise with all possible positive integers $n$ and $k$. These poles are absorbed by the counterterms $\left(Z_{D}-1\right) u$. Thus, the general form of the renormalization factor $Z_{D}$ reads

$$
\begin{aligned}
Z_{D} & =1+Y^{(1)}+O\left(\delta^{-2}\right), \\
Y^{(1)} & =\sum_{n=1}^{\infty} \sum_{k=0}^{n+1}\left(\begin{array}{c}
n+1 \\
k
\end{array}\right) \frac{n C_{n, k}}{-n \varepsilon+2 k \rho} u^{n} w^{k} .
\end{aligned}
$$


In general, the coefficients $C_{n, k}$ may be functions of $\varepsilon$ and $\rho$, depending on the renormalization description. In the minimal renormalization scheme, these coefficients are defined to be independent of $\varepsilon$ and $\rho$. This procedure cancels all simple pole singularities arising in the limit $\varepsilon, \rho \rightarrow 0$ as long as $\rho$ is at least of the same order as $\varepsilon$. One can readily compute these coefficients, which are now independent of $\varepsilon$ and $\rho$, from the model without the correlated noise in the following way. First, consider the Bethe-Salpeter equation (4.2) for $w=0$, i.e. with short-range correlation only. One can easily show that in this case $\left.\Gamma_{2,2}\right|_{w=0}=: \bar{\Gamma}_{2,2}$ is independent of $\mathbf{k}$ and $\mathbf{k}^{\prime}$, and the solution of the BetheSalpeter equation (4.2) is given by

$$
\bar{\Gamma}_{2,2}=\frac{-D g^{2}}{\bar{Z}_{D}^{-1}-\frac{g^{2}}{4} \int_{p}\left(p^{2}+\mu^{2}\right)^{-1}},
$$

which is minimally renormalized exactly by means of

$$
\bar{Z}_{D}=\frac{1}{1-u / 2 \varepsilon}
$$

where $q^{2}=A_{d}^{-1} u \mu^{-\varepsilon}$. Now take the Bethe-Salpeter equation (4.2) in the limit $\rho \rightarrow 0$. This case can be mapped to the preceding one with $w=0$ by setting $u \rightarrow \bar{u}=(1+w) u$ and

$$
\left(\left.Z_{D}(u, w)\right|_{\rho=0}+w\right) u=\bar{Z}_{D}(\bar{u}) \bar{u}
$$

From this equation we get

$$
\left.Z_{D}(u, w)\right|_{\rho=0}=1+\frac{(1+w)^{2} u}{2 \varepsilon}+O\left(\varepsilon^{-2}\right)
$$

from which we infer via comparison with the expansion (4.20), and from $C_{n, k}=$ const. for $\rho \neq 0$,

$$
Z_{D}(u, w)=1+\frac{u}{2}\left(\frac{1}{\varepsilon}+\frac{2 w}{\varepsilon-2 \rho}+\frac{w^{2}}{\varepsilon-4 \rho}\right)+O\left(\delta^{-2}\right)
$$

The calculation of the logarithmic derivatives of the $Z$ factors requires only the simple-pole contributions displayed in Eq. (4.25). With $Z Z_{D}=1$ we eventually arrive at

$$
\begin{aligned}
& \gamma=-\gamma_{D}=-\frac{1}{2}(1+w)^{2} u, \quad \zeta=-\gamma-\gamma_{D}=0 \\
& \beta_{u}=(\varepsilon+\gamma) u, \quad \beta_{w}=-(2 \rho+\gamma) w
\end{aligned}
$$

Note that these renormalization-group functions are exact within the minimal renormalization scheme.

\subsection{Scaling behavior in the smooth phase and at the roughening transition}

We are now in a position to discuss the flow equations (2.37) with the minimally renormalized RG beta functions (4.3) and (4.4), valid to all orders in perturbation theory with respect to the non-linear couplings $u$ and $v$.

\subsubsection{Exact results and flow diagrams}

For $d<d_{c}=2(1+\rho)$, i.e., $\varepsilon=d-2<2 \rho$, Eq. (4.4) implies that $v(\ell) \rightarrow \infty$ as $\ell \rightarrow 0$, see Fig. 4.3(a). Thus in this situation, minimally renormalized perturbation theory (in the representation using the fields $n$ and $\tilde{n}$ ) breaks down, and one must resort to the fixed-dimension expansion discussed above, or an expansion near some other special point in the $(\rho, d)$-plane. For instance, in the particular case $\rho=1$, which represents the Burgers equation with non-conserved noise, an $\epsilon=4-d$ expansion in the vicinity of the upper critical dimension 4 can be constructed [15.

On the other hand, for $d>d_{c}=2(1+\rho)$, the RG flow equation shows that $v(\ell) \rightarrow 0$ in the asymptotic infrared limit $\ell \rightarrow 0$. Hence the usual short-range noise KPZ scenario comes into play [15,2, 6, 36]. As depicted in Fig. 4.3(b), in addition to the trivial fixed point $u=$ 0 and the perturbatively non-accessible strong-coupling fixed point $u_{*}=\infty$, there appears an unstable non-trivial fixed point

$$
u_{c}=2 \varepsilon, \quad v_{c}=0
$$

There is now a separatrix $v_{c}(u)$, depicted as a thick solid line in Fig. 4.3(b), which marks the location of a nonequilibrium phase transition. Eq. (4.2) quite generally implies $z=2$ in the minimal renormalization scheme, and furthermore the existence of the $O(\varepsilon)$ fixed point (4.27) guarantees the validity of the scaling relation (2.45). Thus, we find the following critical exponents at the roughening transition, valid to all orders in perturbation theory $55,39,56$

$$
\eta_{c}=2-d<0, \quad \chi_{c}=0, \quad z_{c}=2 .
$$

Furthermore, we evaluate the stability matrix

$$
\begin{aligned}
\Omega(u, v) & =\left(\begin{array}{ll}
\partial \beta_{u} / \partial u & \partial \beta_{u} / \partial v \\
\partial \beta_{v} / \partial u & \partial \beta_{v} / \partial v
\end{array}\right) \\
& =\left(\begin{array}{cc}
\varepsilon-(u+v) & -(u+v) \\
0 & \varepsilon-2 \rho
\end{array}\right)
\end{aligned}
$$

at the critical fixed point 4.27$)$. For $\Omega(2 \varepsilon, 0)$, we find the negative eigenvalue $-\phi_{c}$, which defines a crossover exponent

$$
\phi_{c}=\varepsilon=d-2
$$

or inverse correlation length exponent $\nu_{c}=\phi_{c}^{-1}$ associated with the second-order dynamic phase transition at $u_{c}=g_{c}^{2}$ [36]. The second eigenvalue of $\Omega(2 \varepsilon, 0)$ is positive,

$$
\omega_{c}=\varepsilon-2 \rho=d-2(1+\rho),
$$

and can be interpreted as a correction-to-scaling exponent associated with the irrelevant coupling $v$. Again, Eqs. (4.30 and (4.31) constitute exact results.

From these exact results, we can determine the upper critical dimension of the roughening transition, at least within the $(2+\varepsilon)$ expansion. In the directed-polymer 

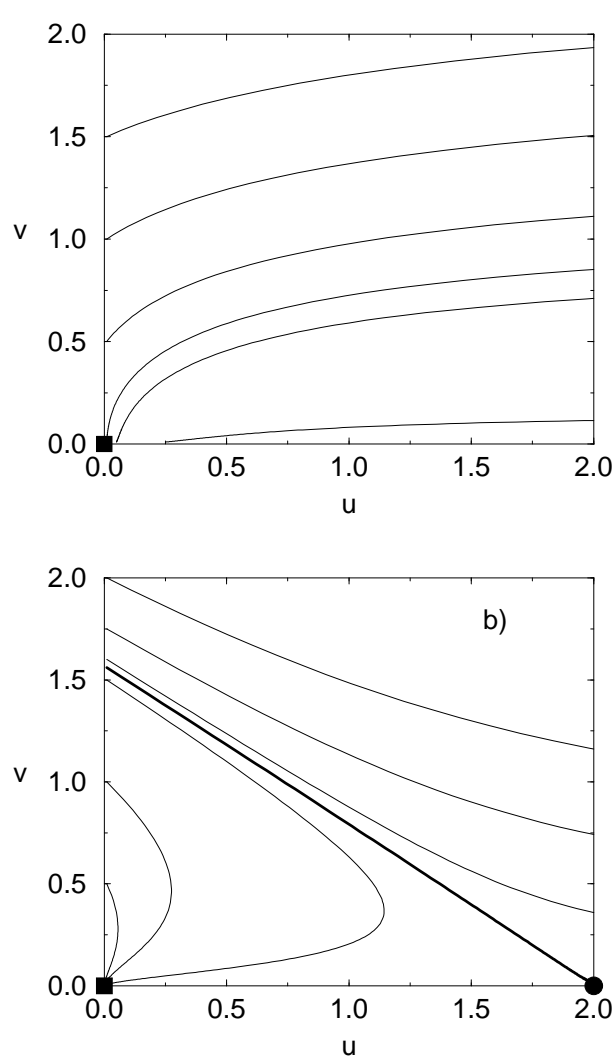

Fig. 4.3. Flow diagram for the KPZ equation with spatially long-range correlated noise for (a) $d=2$ and $\rho=0.2$ : the RG flow for $v$ diverges, and perturbation theory breaks down; (b) $d=3$ and $\rho=0.2$ : the long-range coupling $v \rightarrow 0$ under scale transformations, and the unstable short-range fixed point $u_{c}=2 \varepsilon$ separates a smooth phase $(u \rightarrow 0)$ from a strongcoupling rough phase $(u \rightarrow \infty)$.

representation, the non-equilibrium roughening transition maps onto a second-order equilibrium transition separating a phase where the quenched point defects have very little effect on the polymer structure, from a disorderdominated ("glassy") phase. It is generally accepted that for such a transition the Chayes-Fisher bound [57] $\nu>$ $2 / d$ applies. Assuming that this is valid for the above crossover length scale as well, and furthermore that there appear no non-perturbative corrections in higher dimensions, inspection of Eq. (4.30) tells us that the correlation length exponent reaches its lower bound $\nu_{\min }=2 / d$ at four dimensions, where it then acquires its presumable mean-field value $\nu_{c}^{\mathrm{mf}}=\frac{1}{2}$. Hence the upper critical dimension for the roughening transition is $d_{u c}=4$.

\subsubsection{Scaling behavior in the smooth phase}

Let us now discuss the scaling behavior in the smooth phase. We have seen that for small initial values of $v$ and $u>u_{c}$, the RG flow leads into the strong-coupling regime, which has hitherto resisted any successful analytic approaches. Numerical simulations have found $\chi>0$, and one therefore expects this phase to be characterized by a rough interface. In the converse situation, $u<u_{c}$, both non-linear couplings $u$ and $v$ flow to zero, see Fig. 4.3(b), which renders the scaling relation (2.45) as well as (2.46) invalid. In order to obtain the correct scaling behavior in the ensuing smooth phase, a more detailed investigation of the flow of the ratio $w=v / u$ is required. In terms of the variables $u$ and $w$, the gamma and beta functions become

$$
\begin{aligned}
\gamma & =-\frac{u}{2}(1+w)^{2}, \\
\beta_{u}(u, w) & =u\left[\varepsilon-\frac{u}{2}(1+w)^{2}\right], \\
\beta_{w}(u, w) & =w\left[-2 \rho+\frac{u}{2}(1+w)^{2}\right] .
\end{aligned}
$$

Consequently, as $\ell \rightarrow 0$ and $u(\ell) \rightarrow 0, w(\ell) \rightarrow \infty$.

At this point it is important to note that $w$ appeared as a prefactor in the noise correlation, and therefore multiplies $\tilde{s}^{2}$ in the action (2.19). The divergence of $w$ therefore implies that in order to obtain the correct scaling exponent $\eta$, we need to rescale the fields $\tilde{s}$ and $s$ according to

$$
\tilde{s}^{\prime}=(1+w)^{\frac{1}{2}} \tilde{s}, \text { and } \quad s^{\prime}=(1+w)^{-\frac{1}{2}} s
$$

The multiplicative factor $1+w$ then induces an additional term in the relation between the static exponent $\eta$ and the fixed-point value of $\gamma$, namely

$$
\gamma^{\prime}=\gamma+\frac{\beta_{w}}{1+w}, \quad \eta=\gamma^{\prime *} .
$$

At the roughening transition, $w \rightarrow 0$, and $\eta_{c}=\gamma^{*}=-\varepsilon$ is recovered. In the smooth phase, where $w \rightarrow \infty$ and $v \rightarrow 0$, one obtains, however,

$$
\eta_{\mathrm{sm}}=-2 \rho .
$$

With $\zeta=0$ and Eq. (2.44), we arrive at the scaling exponents in the smooth phase

$$
\chi_{\mathrm{sm}}=1+\rho-\frac{d}{2} \leq 0, \quad z_{\mathrm{sm}}=2 .
$$

Notice that the roughness exponent is negative, which describes a smooth interface, and furthermore that its actual value is determined by the power-law correlated noise. When $\rho=(d-2) / 2$, the long-range noise is prepared in such a way that the roughness exponents in the smooth phase and at the roughening transition coincide, $\chi_{\mathrm{sm}}=\chi_{c}=0$. This special degenerate situation corresponds to $d=d_{c}$, and therefore the scaling behavior in the smooth regime cannot be distinguished from the critical behavior; accordingly, the coupling $v(\ell)$ is constant, see Eq. (4.4). In the flow diagram, we should thus find a fixed line for $d=d_{c}$, compare Fig. 4.5(b) below.

We shall now analyze the flow equations in more detail, and determine the correction-to-scaling exponents $\omega_{1}$ and $\omega_{2}$. The latter are given by the eigenvalues of the stability matrix (4.29), evaluated in the smooth phase, and must both be positive. We shall thus uncover an additional 
subtlety in the correlated-noise KPZ problem, namely the emergence of two distinct smooth regimes characterized by different correction-to-scaling exponents. To this end, we introduce the following new variables

$$
x=\frac{w}{1+w}, \quad y=\frac{1}{4 \rho}(1+w)^{2} u .
$$

The parameter $x$ varies in the interval $[0,1]$ and describes the relative strength of the short-range and power-law correlated noise contributions. The variable $y$ basically coincides with the short-range coupling $u$ at the uncorrelated noise fixed point, where $w=0$, and furthermore represents the appropriate combination in the long-range regime. In addition, it is convenient to re-parametrize the RG flow according to

$$
\ell=e^{-l / 2 \rho}, \quad \ell \frac{d}{d \ell}=-2 \rho \frac{d}{d l} .
$$

In terms of this new variable, the RG flow equations for $x$ and $y$ become

$$
\begin{aligned}
& \frac{d x}{d l}=x(1-x)(1-y), \\
& \frac{d y}{d l}=y\left[1-\frac{\varepsilon}{2 \rho}+(2 x-1)(1-y)\right],
\end{aligned}
$$

and the stability matrix reads

$$
\begin{aligned}
& \Omega(x, y)= \\
& \quad\left(\begin{array}{cc}
-(2 x-1)(1-y) & -x(1-x) \\
2 y(1-y) & -\frac{\varepsilon}{2 \rho}+2 x-2(2 x-1) y
\end{array}\right) .
\end{aligned}
$$

The flow equations (4.41) and (4.42) have four fixed points: (i) $(x, y)=(0,0)$ : Edwards-Wilkinson fixed point, with scaling exponents $\chi=1-\frac{d}{2}$, and $z=2$. The associated eigenvalues of $\Omega(x, y)$ are 1 and $-\varepsilon / 2 \rho$, and this Gaussian fixed point is thus unstable with respect to the long-range noise correlations ( $x$ direction) for $d>2$.

(ii) $(x, y)=(0, \varepsilon / 2 \rho)$ : short-range $\mathrm{KPZ}$ fixed point, with stability matrix eigenvalues $1-\varepsilon / 2 \rho$ and $\varepsilon / 2 \rho$. This fixed point is unstable in both directions for $d<d_{c}$, and describes the roughening transition for $d>d_{c}=2(1+$ $\rho)$, with the critical exponents 4.28 . The crossover and correction-to-scaling exponents (4.30) and (4.31) follow from the above eigenvalues through multiplication with the factor $-2 \rho$, which originates from the re-parametrization (4.40).

(iii) $(x, y)=(1,0)$, with eigenvalues -1 and $2-\varepsilon / 2 \rho$ : unstable for $d<2(1+2 \rho)$, stable for $d>2(1+2 \rho)$.

(iv) $(x, y)=(1,2-\varepsilon / 2 \rho)$, with eigenvalues $1-\varepsilon / 2 \rho$ and $-(2-\varepsilon / 2 \rho)$ : unstable for $d<d_{c}$ and $d>2(1+2 \rho)$, stable for $2(1+\rho)<d<2(1+2 \rho)$.

The fixed points (iii) and (iv) therefore describe the smooth phase with scaling exponents (4.38) in the regimes $d>$ $2(1+2 \rho)$ and $d_{c}=2(1+\rho)<d<2(1+2 \rho)$, respectively, which are characterized by different correction-to-scaling exponents.

To summarize, we have to distinguish between four distinct regimes, as depicted in the $(\rho, d)$-plane in Fig. 4.4.

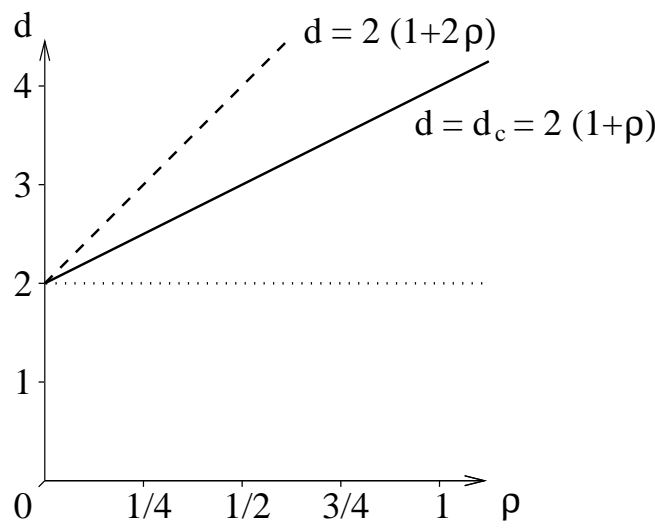

Fig. 4.4. Different regimes for the long-range correlated noise $\mathrm{KPZ}$ problem in the $(\rho, d)$-plane.

(a) For $d<d_{c}=2(1+\rho)$, there exists no non-equilibrium phase transition from a smooth into a rough phase; the RG flow runs away into the strong-coupling regime $y \rightarrow \infty$. To what extent this situation is accessible via renormalized perturbation theory, is to some extend still an open question and will be further discussed in the final section 6.

(b) Precisely at the critical dimension $d=d_{c}$, or $\rho=(d-$ 2 ) $/ 2$, there appears a fixed line at $y=1$, which is unstable for $x<\frac{1}{2}$, and stable for $x>\frac{1}{2}$. The RG trajectories are hyperbolae $y=C / x(1-x)$. The flow separatrix is $x<\frac{1}{2}: y=1, x>\frac{1}{2}: y=1 / 4 x(1-x)$.

Above the critical dimension $d_{c}$, there appears a nonequilibrium transition separating a long-range noise dominated smooth phase from a perturbatively non-accessible strong-coupling rough phase. This roughening transition is described by a separatrix in the flow diagram, terminating at the short-range $\mathrm{KPZ}$ fixed point $(x, y)=(0, \varepsilon / 2 \rho)$. (c) For $d_{c}=2(1+\rho)<d<2(1+2 \rho)$, the stable fixed point characterizing the smooth phase is $(x, y)=(1,2-\varepsilon / 2 \rho)$, with associated correction-to-scaling exponents

$$
\omega_{1}=d-2(1+\rho), \text { and } \omega_{2}=2(1+2 \rho)-d .
$$

(d) For $d \geq 2(1+2 \rho)$, the stable fixed point governing the smooth phase becomes $(x, y)=(1,0)$, characterized by

$$
\omega_{1}=d-2(1+2 \rho) \text {, and } \omega_{2}=2 \rho .
$$

The strong-coupling phase with presumably positive roughness exponent cannot be addressed with our methods. However, the numerical solutions of the flow equations would suggest that $x \rightarrow 0$ in the rough phase, and hence that the long-range noise correlations are irrelevant in the strong-coupling regime. Yet, of course we cannot exclude either that there might exist a further crossover line separating this short-range regime from another scaling region, where the initial long-range noise correlations dominate.

\section{General spatial noise correlations}

We can readily generalize our analysis of the KPZ equation (2.5) to arbitrary spatial noise $R_{0}\left(\mathbf{x}-\mathbf{x}^{\prime}\right)$, provided 

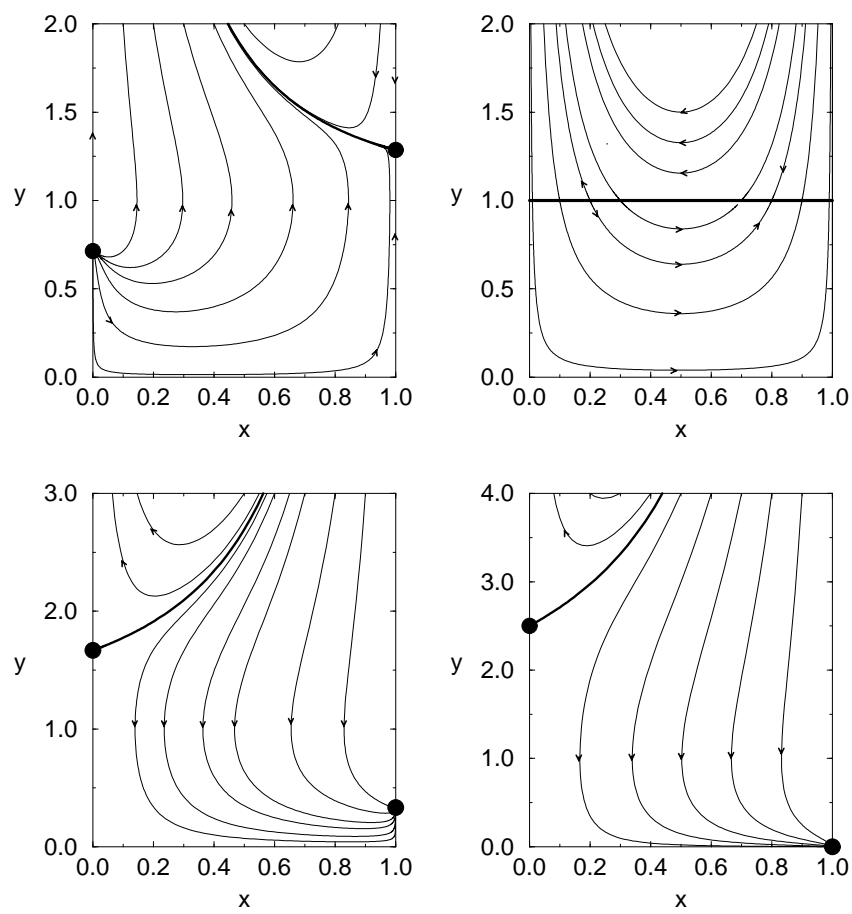

Fig. 4.5. Flow diagrams in terms of the variables $x$ and $y$ in the regimes (a) $d<d_{c}=2(1+\rho)$, (b) $d=d_{c}$, (c) $2(1+\rho)<$ $d<2(1+2 \rho)$, and $(\mathrm{d}) d \geq 2(1+2 \rho)$.

the correlations of the stochastic forces remain local in time, where from now on we indicate the bare form of the noise correlations by an index " 0 ". As of course the structure of the ensuing field theory has not changed as compared to Sec. 1, there still is no propagator renormalization, and therefore $z=2$. Upon rescaling the vertex function $\Gamma_{22}\left(\mathbf{k}, \mathbf{k}^{\prime} ; \mu^{2}\right)$ by a factor $g^{2} / D$, the Bethe-Salpeter equation for the "renormalized" noise reads

$$
\begin{aligned}
& R\left(\mathbf{k}, \mathbf{k}^{\prime} ; \mu^{2}\right)=R_{0}\left(\mathbf{k}-\mathbf{k}^{\prime}\right) \\
& \quad+\frac{g^{2}}{4} \int \frac{d^{d} p}{(2 \pi)^{d}} \frac{R_{0}(\mathbf{k}-\mathbf{p})}{p^{2}+\mu^{2}} R\left(\mathbf{p}, \mathbf{k}^{\prime} ; \mu^{2}\right) .
\end{aligned}
$$

Note that this exact equation allows us to determine the full noise correlation function $R\left(\mathbf{k}, \mathbf{k}^{\prime} ; \mu^{2}\right)$ for any given bare noise $R_{0}(\mathbf{k})$. For simplicity, in the following we shall restrict ourselves to the case where $\mathbf{k}^{\prime}=0$.

\subsection{Mathematical structure of the Bethe-Salpeter equation}

Upon defining as $f\left(k ; \mu^{2}\right)=R\left(\mathbf{k}, \mathbf{k}^{\prime}=\mathbf{0} ; \mu^{2}\right) / R_{0}(k)$ the ratio of the "renormalized" and the bare noise correlator, and carrying out the angular integrations, the BetheSalpeter equation assumes the form

$$
f\left(k ; \mu^{2}\right)=1+\lambda \int_{0}^{\Lambda} d p K\left(k, p ; \mu^{2}\right) f\left(p ; \mu^{2}\right),
$$

where we have introduced an upper momentum cutoff $\Lambda$, and furthermore defined the integral kernel

$$
K\left(k, p ; \mu^{2}\right)=\frac{p^{d-1} R_{0}(p)}{\left(p^{2}+\mu^{2}\right) R_{0}(k)} \int d \eta R_{0}(|\mathbf{k}-\mathbf{p}|),
$$

with $\eta$ denoting the cosine of the angle between the wave vectors $\mathbf{k}$ and $\mathbf{p}$.

Now the Bethe-Salpeter equation has the structure of a Fredholm integral equation of the second kind. The parameter $\lambda$ in the integral equation,

$$
\lambda=\frac{S_{d}}{8} g^{2},
$$

is proportional to the effective interaction parameter of the $\mathrm{KPZ}$ equation, and the kernel $K\left(k, p ; \mu^{2}\right)$ is a functional of the bare noise correlator $R_{0}(k)$ and completely determined by it. In general, the existence of a solution for a Fredholm integral equation depends on the strength of the interaction parameter $\lambda$. It is known that a perturbative solution in terms of a Neumann series exists for sufficiently small $\lambda$, provided the kernel $K\left(k, p ; \mu^{2}\right)$ is bounded. Note that the von-Neumann series is nothing but the perturbation expansion symbolically depicted in Fig. 1.2. The critical value $\lambda_{c}$, where a perturbative solution breaks down, is given by the eigenvalue of the homogeneous equation $f\left(k ; \mu^{2}=0\right)=\lambda \int_{0}^{\Lambda} d p K\left(k, p ; \mu^{2}=0\right) f\left(p ; \mu^{2}=0\right)$. Physically, this eigenvalue corresponds to the critical parameter value of the effective coupling constant where a phase transition from the smooth to the rough phase occurs. Given a certain functional form of the bare noise correlator, this allows for an exact determination of the location of the roughening transition, i.e., the structure of the phase diagram. In this way, we can lucidly relate the mathematical properties of the Bethe-Salpeter or Fredholm integral equation to the physical structure emerging by means of the renormalization-group analysis.

Formally, there are also solutions of the Fredholm integral equation above the critical value of the $\lambda$-parameter which cannot be obtained in the form of a von-Neumann series (perturbation expansion). From the numerical solutions (see below), it turns out that the noise correlations then become negative in a certain wave-vector regime. The physical significance of these regions of negative correlations is unclear, and it would be mere speculation to assign any meaning to them associated with the strong-coupling phase.

\subsection{Numerical solution of the Bethe-Salpeter equation}

In this section, we study the numerical solution of the Bethe-Salpeter integral equation for $\mu^{2}=0$ and two different forms of the wave-vector dependence for the bare noise correlation function, namely (i) Gaussian, and (ii) power-law correlated noise. 


\subsubsection{Gaussian noise}

We start with a bare noise correlation function given by a Gaussian

$$
R_{0}(k)=\exp \left(-\alpha k^{2}\right) .
$$

Then the integral kernel takes the form

$$
K(k, p)=\frac{p^{d-4}}{2 k} e^{-2 \alpha p^{2}} \sinh (2 \alpha k p) .
$$

The ensuing integral equation may be solved by means of standard numerical procedures, leading to the results for the renormalized noise correlator $R(k)=f(k) R_{0}(k)$ shown in Fig. 5.1 in three dimensions, and with noise parameters $\alpha=5, \Lambda=3$ and interaction parameters $\lambda=0$, $0.5,1.0$ and 1.5 , respectively. It is seen that upon increasing $\lambda$ towards ist critical value of $\lambda_{c} \approx 1.515$, the amplitude of the renormalized noise at wave vector $k=0$ diverges as a power law with an exponent given by approximately -1 .

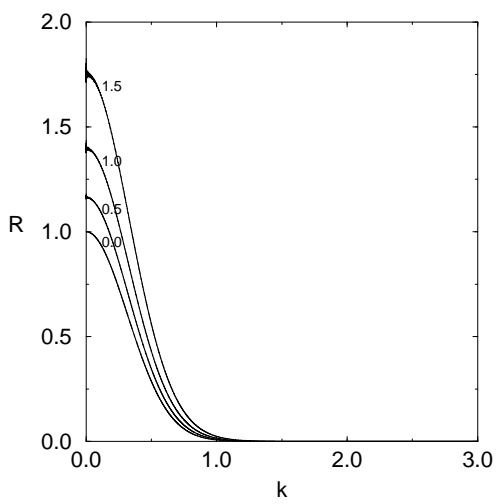

Fig. 5.1. Renormalized noise correlator $R(k)=f(k) R_{0}(k)$ calculated from the Bethe-Salpeter equation in $d=3$ with a bare Gaussian noise $R_{0}(k)=\exp \left(-5 k^{2}\right)$ for values $\lambda=0,0.5$, 1.0 and 1.5 of the interaction parameter (as indicated in the graph). The cutoff was fixed to $\Lambda=3$.

\subsubsection{Power law noise}

Next we consider the solution of the Bethe-Salpeter equation starting from a bare power law noise of the form

$$
R_{0}(k)=k^{-2 \rho} .
$$

Then the integral kernel becomes

$K(k, p)=\frac{k^{2 \rho-1} p^{d-4-2 \rho}}{2(1-\rho)}\left[(k+p)^{2(1-\rho)}-(k-p)^{2(1-\rho)}\right]$.

With increasing interaction parameter, the spectrum of the noise correlator shows an overall increase, but at the same time it develops more weight at wave vectors close to the cutoff $\Lambda$. In Fig. 5.2, a solution of the Bethe-Salpeter equation is shown for the parameter values $\rho=\frac{1}{4}$ and $\Lambda=3$ in $d=3$. Again, there is a critical value $\lambda_{c}$ where the noise amplitude shows a power law divergence.

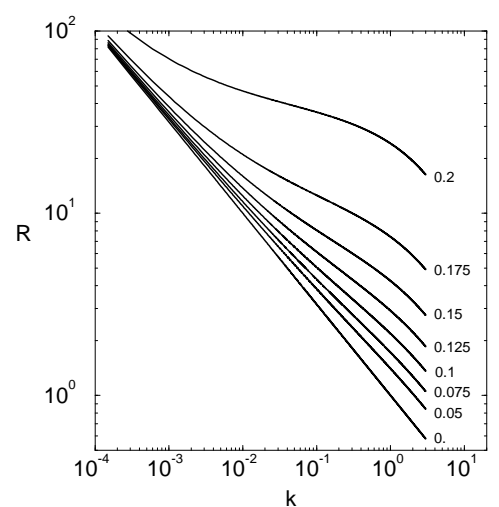

Fig. 5.2. Renormalized noise correlator $R(k)=f(k) R_{0}(k)$ calculated from the Bethe-Salpeter equation in $d=3$ with a power law noise $R_{0}(k)=k^{-\frac{1}{2}}$ for values $\lambda=0,0.05,0.075$, $0.1,0.125,0.15,0.175$ and 0.2 of the interaction parameter (as indicated in the graph). The cutoff was fixed to $\Lambda=3$.

The two particular forms of the bare noise correlation functions discussed above may exemplify the potential of the Bethe-Salpeter equation in determining the structure of the phase diagram, and specifically, the line in parameter space where the roughening transition occurs. If required (e.g., for a particular numerical simulation) precise values could be determined allowing for quite stringent tests on the theoretical concepts presented in this paper.

\section{Summary and discussion}

In this paper, we have studied the Kardar-Parisi-Zhang equation with long-range correlated noise (1.2). Our results are best summarized in the following "phase diagram" (Fig. 6.1) in the plane spanned by the spatial dimension $d$ and the correlation exponent $\rho$ of the noise. Here, the solid line defined by $d=d_{c}=2(1+\rho)$ indicates the exactly determined boundary line above which the Kardar-Parisi-Zhang equation with correlated noise shows a non-equilibrium roughening transition, separating a Gaussian (EW) smooth regime from a strong-coupling rough phase, and below which there exists only a rough phase.

\subsection{Exact results for the roughening transition above the lower critical dimension}

We start our summary with a discussion of the results above the lower critical dimension $d_{c}$. Here, there are two 


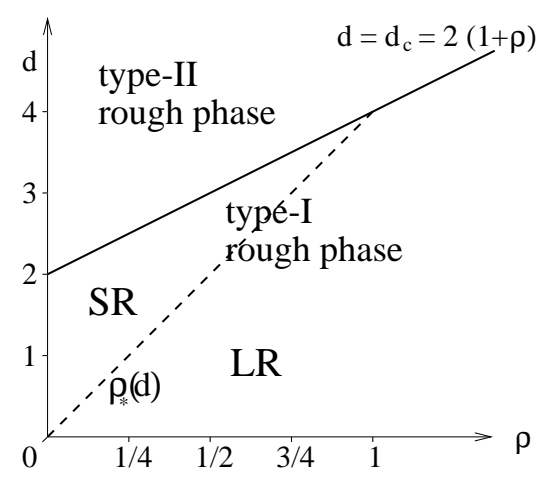

Fig. 6.1. "Phase diagram" for the KPZ equation with correlated noise in the $(\rho, d)$ plane. The solid line, $d=d_{c}=2(1+\rho)$, indicates the exactly determined boundary line above which the Kardar-Parisi-Zhang-equation with correlated noise shows a non-equilibrium phase transition, and below which there exists only a rough phase.

stable and one unstable (critical) fixed points, describing a smooth and rough phase, respectively, as well as the non-equilibrium separating these. Using a stochastic ColeHopf transformation, we were able to derive the following exact results for the roughening transition and the scaling properties in the smooth phase:

a. The roughening transition is characterized by an unstable fixed point much in the same way as the temperature-driven phase transition in the non-linear $\sigma$-model. As a result, which is exact to all orders in a $(2+\varepsilon)$ expansion, we obtain for the dynamic exponent $z_{c}=2$, the roughness exponent $\chi_{c}=0$, the crossover exponent $\phi_{c}=d-2$, and the correction-to-scaling exponent $\omega_{c}=d-2(1+\rho)$. While $\phi_{c}$ describes the divergence of the crossover length scale, $\omega_{c}$ governs the next-toleading terms in the corresponding scaling functions.

b. For effective coupling constants less than the values corresponding to the unstable fixed point, the flow tends towards a stable fixed point that describes the smooth phase. We find that the long-range fixed point is stable, and that the smooth phase is described by the scaling exponents $z_{\mathrm{sm}}=2, \chi_{\mathrm{sm}}=1+\rho-d / 2$, and the correction-to-scaling exponents $\omega_{1}=d-2(1+\rho)$, $\omega_{2}=2(1+2 \rho)-d$ for $2(1+\rho)<d<2(1+2 \rho)$, and $\omega_{1}=d-2(1+2 \rho), \omega_{2}=2 \rho$ for $d \geq 2(1+2 \rho)$, respectively.

c. The flow of the RG trajectories starting with effective couplings larger than the critical value, tends to infinity and seems to converge towards short-range noise behavior. Although we cannot exclude that the flow returns to effectively long-range noise behavior at larger couplings, this result seems to indicate that long-range noise does not affect the critical behavior of the strongcoupling rough phase above $d_{c}$.

d. For any given initial ("bare") noise correlator that is local in time, the noise correlation function can be determined exactly using a Bethe-Salpeter integral equation which relates the bare to the full noise correlation.
The mathematical structure of this Fredholm integral equation reflects the renormalization group results.

The rough phase above $d_{c}$ does not seem to be accessible by any perturbational means, and is presumably qualitatively different from the rough phase below $d_{c}$, which we are going to discuss next.

\subsection{Exact results and some conjectures on the rough phase below the lower critical dimension}

In dimensions $d<d_{c}=2(1+\rho)$, the interface scaling behavior is governed by a strong-coupling fixed point. Contrary to the case above $d_{c}$, there actually do exist some perturbational techniques which allow for the assessment of the ensuing scaling behavior:

a. First of all — as was already noted in Ref. [6] the general structure of the RG theory tells us that there are two possible fixed points, one corresponding to short-range noise, the other one to long-range noise. In addition, if the long-range noise fixed point becomes stable, the critical exponents here can be read off from the RG equation exactly, without knowing the actual value of the fixed point. One obtains

$$
\chi_{\mathrm{lr}}=\frac{2-d+2 \rho}{3} \text {, and } z_{\mathrm{lr}}=\frac{4+d-2 \rho}{3} .
$$

b. One way to approach the strong-coupling behavior below $d_{c}$ is by means of a non-minimal renormalization scheme. In one dimension, we establish the exact result that the usual KPZ short-range noise fixed point with scaling exponents $\chi_{\mathrm{sr}}=\frac{1}{2}$ and $z_{\mathrm{sr}}=\frac{3}{2}$ remains stable, provided $\rho<\frac{1}{4}$. For $\frac{1}{4} \leq \rho<1$, we find $\chi_{\mathrm{lr}}=(2-d+2 \rho) / 3$ and $z_{\mathrm{lr}}=(4+d-2 \rho) / 3$. These results may also be re-derived within a proper $\varepsilon$ expansion using a mapping to driven diffusive systems [41.

c. In the $(\rho, d)$-plane, the point $(1,4)$ is a special point since it describes the Burgers equation with a non-conserved noise, i.e., model B in the pioneering work by Forster et al. [15]. As we have already noted previously in this paper and as is explicitly shown in Ref. [15], the infrared behavior of the non-conserved Burgers equation is governed by an infrared-stable (long-range noise) fixed point which can be explicitly obtained within a one-loop perturbative calculation around the upper critical dimension $d_{\mathrm{uc}}=4$ of this model. Actually, the dynamic exponent obtained via the $\varepsilon$ expansion turns out to be the same as the one resulting from exact arguments (see item a. above), namely $z_{\mathrm{lr}}=(2+d) / 3$.

In the remainder of this section, we discuss the boundary line between the short-range and long-range noise fixed points below the lower critical dimension $d_{c}$. In order to determine the location of this boundary line, we only need to return to the above results, and combine them with established facts from the literature. First, we know from the exact solution of the KPZ equation in $d=1$ that 
the critical value of $\rho$ above which the short-range noise fixed point becomes unstable is $\rho=\frac{1}{4}$. Next, we may ask where the boundary between the short-range and longrange fixed points in the $(\rho, d)$-plane crosses the line marking the lower critical dimension, $d_{c}=2(1+\rho)$, compare Fig. 6.1. It is actually quite easy to see that this crossing point is given by $(\rho, d)=(1,4)$, simply by noting that for $\rho=1$, we arrive at the non-conserved Burgers equation with associated upper critical dimension $d_{c}=4$. In addition, upon adding noise with $\rho>1$ to the nonconserved noise of the Burgers equation, i.e., at the longrange noise fixed point, the dynamic exponent becomes $z=(4+d-2 \rho) / 3$. Hence it is quite likely that at $d=4$, and for $\rho \rightarrow 1$, this fixed point coincides with the unstable fixed point describing the roughening transition of the KPZ equation, with $z_{c}=2$ and $\phi_{c}=\nu_{c}^{-1}=2$ - in fact, all of these fixed points become zero at this very particular point in the $(\rho, d)$-plane. Notice that this essentially excludes the other possible guess, namely that the longrange noise fixed point from the regime below $d_{c}$ turns into the strong-coupling fixed point above the roughening transition at $(\rho, d)=(1,4)$. Of course, one might still argue that this strong-coupling fixed point vanishes as well at this point, but this would appear rather strange.

Having established that the separatrix between the long-range and short-range noise fixed points has to go through two points, $(\rho, d)=\left(\frac{1}{4}, 1\right)$ and $(1,4)$, we would also like to know the location of this curve in between. As a crude approximation to the actual functional form of the separatrix $\rho_{*}(d)$, one may take a linear interpolation between the two exactly determined points, i.e., $\rho_{*}(d)=d / 4$. In fact, recent computer simulations 24 found $\rho_{*}(d=2) \approx 0.5$, which confirms that this simple interpolation formula may already be quite close to the actual function $\rho_{*}(d)$. Note that the knowledge of the separatrix enables us to determine the critical exponents of the short-range noise strong-coupling fixed point, if one makes the quite plausible assumption that the critical exponents are continuous functions of $\rho$. Inserting the linear interpolation formula into the exact value for the longrange dynamic exponent, this would give

$$
z=\frac{4+d-2(d / 4)}{3}=\frac{8+d}{6}
$$

Interestingly, this simple estimate turns out be identical with the result obtained from a functional renormalization group analysis by Halpin-Healy [17, and a more recent mode-coupling study by Bhattacharjee [58]; and of course, it reproduces the exact one-dimensional value $z=3 / 2$.

What can we learn from this? If we assume that the critical exponents are really continuous over the whole parameter range of $\rho$ and $d$, this would imply that we would actually know the values of the critical exponents in the rough phase, both below and above the lower critical dimension $d_{c}$. As an immediate consequence, this would tell us that 4 is the upper critical dimension for both the rough phase and the roughening transition in accordance with some recent speculations 59. However, because of the divergence of the strong-coupling fixed point in the short-range KPZ equation at $d_{c}=2$, which indicates non-analytic behavior at the lower critical dimension [36 39,38], we do not think that this assumption is correct. Instead, we suggest the following scenario:

a. One has to distinguish between two fundamentally different notions of rough phases, one below the lower critical dimension $d_{c}=2(1+\rho)$, and the other one above; in the following, we shall denote these two distinct rough phases as type-I and type-II, respectively.

b. The dashed line in Fig. 6.1 marks the separatrix between the short-range and long-range noise fixed point of the type-I rough phase below the lower critical dimension. The critical exponents are continuous at this line.

c. Anticipating that the separatrix between long-range and short-range noise fixed points is approximately given by $\rho=d / 4$, the scaling exponents in the sliver below the solid line marking the lower critical dimension $d_{c}(\rho)$ of the problem become

$$
z=\frac{d+8}{6}, \quad \text { and } \quad \chi=2-z .
$$

d. It is possible to assess the scaling behavior in this typeI regime in various ways. First of all, exact relations tell us that once we are below the dashed line, i.e., asymptotically at the long-range fixed point, all exponents are known exactly 60]. In addition, a perturbative expansion may be performed for the non-conserved Burgers equation near the upper critical dimension 4 of this model. In the regime where the short-range noise fixed point becomes stable, several approaches have turned out to be useful: (i) a fixed dimension RG approach outlined in section [3, see also Ref. [36]; (ii) modeoupling theory in $d=1$ [61,62]; and (iii) a perturbative mode-coupling approach near $d=4$ [58]. Furthermore, it seems that the functional RG approach by HalpinHealy [17] also applies in this regime, since it yields the identical result for the dashed line as our linear interpolation between two exactly known results. It would be interesting to better understand the apparent relationship between the mode-coupling and functional RG approaches, which is suggested by the above results. It is important to note at this point that whenever one allows, even implicitly, for long-range correlated noise in the KPZ problem, the lower critical dimension is shifted upwards. One must then be very careful not to mistake the ensuing type-I strong-coupling scaling behavior with the type-II strong-coupling phase above the roughening transition.

e. As an immediate consequence of the discussion in item d., the upper critical dimension of the type-I rough phase below the lower critical dimension is 4 .

f. The scaling exponents of the rough phase are not continuous at the lower critical dimension. As indicated by the divergence of the effective coupling constant within a fixed-dimension 2-loop calculation [36], there seems to exist a qualitatively new strong-coupling behavior once a roughening transition between a smooth 
and a rough phase appears. The behavior of the typeII rough phase above the lower critical dimension ist most likely not even described in terms of the continuous KPZ model, and the detailed scaling properties might well be non-universal. Recent investigations, where various non-universal aspects of the typeII strong-coupling phase of the short-range KPZ equation are observed 63,64,65,66, tend to support this view.

g. The critical exponent at the roughening transition, as well as the noise correlations, can be obtained exactly by means of a stochastic Cole-Hopf transformation leading to an exact Bethe-Salpeter integral equation for the noise correlation function. The upper critical dimension of the roughening transition is 4 .

In summary, we suggest that the above scenario provides a coherent picture for most of the available numerical and analytical results on the KPZ problem. In particular, it resolves the long-standing discrepancies between the numerical observation that there is no indication of an upper critical dimension near four dimensions, and several recent speculations that despite this fact $d=4$ should be the upper critical dimension of the rough phase. We shall not attempt to judge on the validity of several recent claims in favor of the upper critical dimension 4. Our view presented above would imply that all of these investigations are mainly concerned with the behavior of the type-I rough phase below the lower critical dimension $d_{c}$, shifted upwards as a consequence of the appearance of effectively long-range power-law noise correlations. For the mode-coupling approaches it is clear how this comes about. Here, one starts by construction with long-range correlated noise, and subsequently tries to find a selfconsistent solution. This is likely to almost automatically restrain the mode-coupling approach to the regime below the line marking the lower critical dimension. Hence these methods rather describe the type-I strong-coupling phase rather than the type-II strong-coupling phase they were aiming at.

To our opinion, besides the precise location of the separatrix $\rho_{*}(d)$ below the lower critical dimension $d_{c}$, the truly open issue in the non-equilibrium surface growth problem described by the KPZ equation, thus constitutes the scaling behavior in the type-II strong-coupling phase beyond the non-equilibrium roughening transition that appears for $d>d_{c}$. Future work, probably based on the original discrete growth models, should address the fundamental questions whether, and in which sense there is universality in this regime, and furthermore whether the continous KPZ equation does in fact provide an adequate continuous representation of the ensuing physics.

We benefited from discussions with J. Cardy, T. Halpin-Healy, and K. Wiese. H.K.J. acknowledges support from the SFB 237 ("Unordnung und große Fluktuationen") from the Deutsche Forschungsgemeinschaft (DFG). U.C.T. and E.F. acknowledge support from the Deutsche Forschungsgemeinschaft through a habilitation and a Heisenberg fellowship, respectively, DFGGz. Ta 177 / 2-1,2 and Fr 850 / 3-1.

\section{A Detailed balance conditions in terms of the Fokker-Planck equation}

The validity of detailed balance in $d=1$ dimensions can also be derived in the Burgers representation within the framework of the associated Fokker-Planck equation. The Burgers equation reads

$$
\frac{\partial \mathbf{u}}{\partial t}+\frac{D g}{2} \nabla \mathbf{u}^{2}=D \nabla^{2} \mathbf{u}+\eta
$$

where $\mathbf{u}=-\nabla s$, and for $w=0$

$$
\left\langle\eta_{\alpha}(\mathbf{x}, t) \eta_{\beta}\left(\mathbf{x}^{\prime}, t^{\prime}\right)\right\rangle=-2 D \nabla_{\alpha} \nabla_{\beta} \delta\left(\mathbf{x}-\mathbf{x}^{\prime}\right) \delta\left(t-t^{\prime}\right) .
$$

For $D g=1$, the Langevin equation (A.1) reduces to the noisy Burgers equation. For $g=0$, the stationary distribution for the diffusive field $\mathbf{u}$ with short-range conserved noise is obviously given by

$$
\mathcal{P}_{s t}[\mathbf{u}] \propto \exp \left[-\frac{1}{2} \int \mathbf{u}(\mathbf{x})^{2} d^{d} x\right] .
$$

By writing down the Fokker-Planck equation associated with the stochastic process (A.1), one readily derives the following condition that the stationary distribution (A.3) remain unaltered by the non-linear term $\propto g$ [28],

$$
\begin{aligned}
0 & =\int d^{d} x \frac{\delta}{\delta u(\mathbf{x}, t)}\left(\nabla \mathbf{u}^{2}(\mathbf{x}, t) \mathcal{P}[\mathbf{u}]\right) \\
& =\int d^{d} x\left(2 \nabla \cdot \mathbf{u}-\mathbf{u} \cdot \nabla \mathbf{u}^{2}\right) \mathcal{P}[\mathbf{u}] .
\end{aligned}
$$

While the first term in this expression vanishes, provided appropriate boundary conditions are employed, the second contribution is generally non-zero in any substrate dimension $d \neq 1$. In one dimension, however, it reduces to $\int\left(d u^{3} / d x\right) d x=0$, and (A.3) is indeed the stationary distribution even for the non-linear problem.

\section{B Consequences of Galilean Invariance}

In this appendix, we will exploit the invariance of the dynamic functional $\mathcal{J}$ in the Cole-Hopf representation under a Galilean transformation. The transformation of the fields introduced in (4.5), (4.6) and (2.20) reads to linear order in the parameter $\mathbf{v}$,

$$
\begin{aligned}
& n(\mathbf{x}, t) \rightarrow n(\mathbf{x}+2 D \mathbf{v} t, t) \exp (\mathbf{v} \cdot \mathbf{x}) \\
& \tilde{n}(\mathbf{x}, t) \rightarrow \tilde{n}(\mathbf{x}+2 D \mathbf{v} t, t) \exp (-\mathbf{v} \cdot \mathbf{x}) .
\end{aligned}
$$

Thus the Galilei transformation is represented by a type of gauge transformation with a gauge function linear in $\mathbf{x}$. Including translational invariance, it follows that each invariant term of $\mathcal{J}$ consists of an equal number of $n$ and $\tilde{n}$ fields, because the gauge factors must completely canceled. Therefore, the dynamic functional can be written in the form

$$
\mathcal{J}=\int d t \int d^{d} x\{\tilde{n} \dot{n}+D \nabla \tilde{n} \nabla n-D \mathcal{V}[\tilde{n} n]\} .
$$


In a fully local theory, we can expand with respect to powers of the fields and their derivatives,

$$
\mathcal{V}[\tilde{n} n]=g_{2}(\tilde{n} n)^{2}+g_{3}(\tilde{n} n)^{3}+\cdots+g_{2}^{\prime}(\nabla(\tilde{n} n))^{2}+\cdots .
$$

Below and near $d_{c}=2$, only the first coupling constant $g_{2}=g^{2}$ acquires positive scaling dimension. As usual, this demonstrates the renormalizability of the theory with only $g_{2} \neq 0$, i.e., the original Burgers-KPZ theory, or, in other words, the universality of this theory with respect to a representation of Galilei invariance through a stochastic field theory with one scalar field and its conjugate response field.

For $d>d_{c}=2$, a renormalized perturbation theory can be constructed in analogy with the non-linear $\sigma$-model by means of an $\varepsilon$ expansion with $\varepsilon=d-2>0$. Here, the perturbation theory for the vertex function $\Gamma_{2,2}$ is completely resumable with the aid of the Bethe-Salpeter equation, and leads to exact renormalizations. However, the negative scaling dimension of $g_{2}$ for $\varepsilon>0$, as well as the emergence of higher-order invariants in the expansion of $\mathcal{V}$ in Eq. (B.3), remind us that such a construction is in generally only possible if one lets $\varepsilon \rightarrow 0$ first, and only afterwards the order of the perturbation expansion $N \rightarrow \infty$. This is true because at order $N=O(2 / \varepsilon)$, new primitive divergences are generated which have to be eliminated by counterterms taking the functional form of the higherorder contributions. Luckily, in the case of the BurgersKPZ field theory in the Cole-Hopf representation in the smooth phase, i.e., below the roughening transition, the perturbation theory of the renormalization factors stops already at second order. But it is generally dangerous, for example, to speculate on $d=4$ as a new higher critical dimension by means of purely perturbational arguments only.

Let us now consider the general form of an invariant bi-quadratic term in the dynamic functional $\mathcal{J}$ or in the generating functional $\Gamma$ for the vertex functions,

$$
\mathcal{K}=\int_{x, t} \tilde{n}\left(\mathbf{x}_{1}, t_{1}\right) \tilde{n}\left(\mathbf{x}_{2}, t_{2}\right) K(\{\mathbf{x}, t\}) n\left(\mathbf{x}_{3}, t_{3}\right) n\left(\mathbf{x}_{4}, t_{4}\right)
$$

like the correlated-noise term in $\mathcal{J}$, i.e., the contribution $\Gamma_{2,2}$ to $\Gamma$ in our model. The coupling function $K$ must obey the Galilean invariance condition (to linear order in the parameter $\mathbf{v}$ )

$$
K(\{\mathbf{x}, t\})=K(\{\mathbf{x}+2 D \mathbf{v} t, t\}) e^{\mathbf{v} \cdot\left(\mathbf{x}_{1}+\mathbf{x}_{2}-\mathbf{x}_{3}-\mathbf{x}_{4}\right)} .
$$

Clearly, a fully local interaction or the correlated noise term both proportional at least to $\delta\left(\mathbf{x}_{1}-\mathbf{x}_{3}\right) \delta\left(\mathbf{x}_{2}-\mathbf{x}_{4}\right)$ fulfill Eq. (B.4). After Fourier transformation, we get

$$
\hat{K}(\{\mathbf{p}, \omega\})=\hat{K}(\{\mathbf{p}-i \mathbf{v}, \omega+2 D \mathbf{v} \cdot \mathbf{p}\}),
$$

where, from translational invariance, $\mathbf{p}_{1}+\mathbf{p}_{2}=\mathbf{p}_{1}+\mathbf{p}_{2}=$ : $\mathbf{q}$ and $\omega_{1}+\omega_{2}=\omega_{1}+\omega_{2}=: \omega$. Defining outgoing and ingoing wave vector transfers $\mathbf{k}, \mathbf{k}^{\prime}$ as in Fig. 4.1, accompanied with corresponding frequency transfers $\nu$ and $\nu^{\prime}$, the function $\hat{K}$ can be reduced by Eq. (B.4) to the form

$\hat{K}(\{\mathbf{p}, \omega\})=\hat{K}\left(\mathbf{k}, \mathbf{k}^{\prime} ; i \nu+D \mathbf{q} \mathbf{k}, i \nu^{\prime}+D \mathbf{q k}^{\prime}, 2 i \omega+D \mathbf{q}^{2}\right)$.

Causality shows that $\hat{K}$ is an analytic function of $i \omega$ in the upper complex half-plane, whereas the analytic properties with respect to the variables $\nu, \nu^{\prime}$ are more involved. If $K(\{\mathbf{x}, t\}) \propto \delta\left(t_{1}-t_{2}\right) \delta\left(t_{3}-t_{4}\right)$, the Fourier transform of the function $\hat{K}$ is independent of the frequency transfers. Then Eq. (B.5) implies

$$
\hat{K}(\{\mathbf{p}, \omega\})=\hat{K}\left(\mathbf{k}, \mathbf{k}^{\prime} ; 2 i \omega+D \mathbf{q}^{2}\right) .
$$

Because all terms contributing to the Bethe-Salpeter equation (4.2) display such a time dependence, we find especially

$$
\begin{aligned}
& \Gamma_{2,2}\left(\mathbf{k}, \mathbf{k}^{\prime} ; \frac{2 i \omega}{D}+\mathbf{q}^{2}\right) \\
& =\hat{R}\left(\mathbf{k}-\mathbf{k}^{\prime}\right)+\Gamma_{2,2}^{(D)}\left(\mathbf{k}, \mathbf{k}^{\prime} ; \frac{2 i \omega}{D}+\mathbf{q}^{2}\right)
\end{aligned}
$$

where $\Gamma_{2,2}^{(D)}$ is free of singularities in the limit of vanishing arguments.

\section{References}

1. S. F. Edwards and D. R. Wilkinson, Proc. R. Soc. London A 381, 17 (1982).

2. M. Kardar, G. Parisi, and Y.-C. Zhang, Phys. Rev. Lett. 56, 889 (1986).

3. P. Meakin, Phys. Rep. 235, 189 (1993).

4. T. Halpin-Healy and Y.-C. Zhang, Phys. Rep. 254, 215 (1995).

5. J. Krug, Adv. Phys. 46, 139 (1997).

6. E. Medina, T. Hwa, M. Kardar, and Y.-C. Zhang, Phys. Rev. A 39, 3053 (1989).

7. Y.-C. Zhang, J. Phys. (Paris) 51, 2129 (1990).

8. Z. Csahók, K. Honda, and T. Vicsek, J. Phys. A. 24, L171 (1993).

9. L. A. N. Amaral, A.-L. Barabási, and H. E. Stanley, Phys. Rev. Lett. 73, 62 (1994).

10. J. Krug and P. Meakin, Phys. Rev. Lett. 66, 703 (1991).

11. S. Mukherji and S. M. Bhattacharjee, Phys. Rev. Lett. 79, 2502 (1997).

12. M. Kardar, J. Appl. Phys. 81, 3601 (1987).

13. T. Nattermann, Europhys. Lett. 4, 1241 (1987).

14. Y.-C. Zhang, J. Phys. A. 19, L941 (1986).

15. D. Forster, D. R. Nelson, and M. J. Stephen, Phys. Rev. A 16, 732 (1977).

16. T. Halpin-Healy, Phys. Rev. Lett. 62, 445 (1989).

17. T. Halpin-Healy, Phys. Rev. A 42, 711 (1990).

18. Y.-C. Zhang, Phys. Rev. B 42, 4897 (1990).

19. J. Amar, P.-M. Lam, and F. Family, Phys. Rev. A 43, R4548 (1991).

20. C.-K. Peng, S. Havlin, M. Schwartz, and H. E. Stanley, Phys. Rev. A 44, R2239 (1981). 
21. N. N. Pang, Y. K. Yu, and T. Halpin-Healy, Phys. Rev. E 52, 3224 (1995).

22. F. Hayot and C. Jayaprakash, Phys. Rev. E 54, 4681 (1996).

23. F. Hayot and C. Jayaprakash, Phys. Rev. E 56, 227 (1997).

24. M. S. Li, Phys. Rev. E 55, 1178 (1997).

25. L. Peliti, J. Phys. (Paris) 46, 1469 (1984).

26. B. P. Lee, J. Phys. A. 27, 2633 (1994).

27. B. P. Lee and J. L. Cardy, J. Stat. Phys. 80, 971 (1995).

28. U. Deker and F. Haake, Phys. Rev. A 11, 2043 (1975).

29. P. C. Martin, E. D. Siggia, and H. A. Rose, Phys. Rev. A 8, 423 (1973).

30. H. K. Janssen, Z. Phys. B 23, 377 (1976).

31. C. de Dominicis, J. Phys. (Paris) Colloq. 37, C (1976).

32. H. K. Janssen, in Dynamical Critical Phenomena and related Topics, Vol. 104 of Lecture Notes in Physics, edited by C. P. Enz (Springer Verlag, Berlin, 1979).

33. H. K. Janssen, in From Phase Transitions to Chaos, edited by G. Györgyi, I. Kondor, L. Sasvári, and T. Tél (World Scientific, Singapore, 1992), p. 47.

34. R. Bausch, H. K. Janssen, and H. Wagner, Z. Phys. B 24, 113 (1976).

35. C. D. Dominicis and L. Peliti, Phys. Rev. B 18, 353 (1978).

36. E. Frey and U. C. Täuber, Phys. Rev. E 50, 1024 (1994).

37. This is in complete analogy with the standard procedure in the static $\Phi^{4}$ model, where the prefactor of the $(\nabla \Phi)^{2}$ term is conventionally set to 1 , and its renormalization is absorbed into a non-trivial field renormalization. The ensuing anomalous dimension eventually determines the static critical exponent $\eta$.

38. U. C. Täuber and E. Frey, Phys. Rev. E 51, 6319 (1995).

39. M. Lässig, Nucl. Phys. B448, 559 (1995).

40. H. K. Janssen and B. Schmittmann, Z. Phys. B 63, 517 (1986).

41. H. K. Janssen and B. Schmittmann, unpublished (1998).

42. K. J. Wiese, Phys. Rev. E 56, 5013 (1997).

43. H. K. Janssen (unpublished).

44. K. J. Wiese, e-print: cond-mat/9802068 (1998).

45. J. Sak, Phys. Rev. B 8, 281 (1973).

46. M. A. Gusmão and W. K. Theumann, Phys. Rev. B 28, 6545 (1983).

47. W. K. Theumann and M. A. Gusmão, Phys. Rev. B 31, 379 (1985).

48. J. Honkonen and M. Y. Nalimov, J. Phys. A. 22, 751 (1989).

49. P. Grassberger, Z. Phys. B 47, 365 (1982).

50. D. A. Huse, C. L. Henley, and D. S. Fisher, Phys. Rev. Lett. 55, 2924 (1985).

51. M. Kardar and Y.-C. Zhang, Phys. Rev. Lett. 58, 2087 (1987).

52. D. S. Fisher and D. A. Huse, Phys. Rev. B 43, 10728 (1991).

53. T. Hwa and D. S. Fisher, Phys. Rev. B 49, 3136 (1994).

54. H. Risken, The Fokker-Planck Equation, 2 ed. (Springer, Heidelberg, 1988).

55. C. A. Doty and J. M. Kosterlitz, Phys. Rev. Lett. 69, 1979 (1992).

56. H. K. Janssen, Phys. Rev. Lett. 78, 1082 (1997).

57. J. T. Chayes, L. Chayes, D. S. Fisher, and T. Spencer, Phys. Rev. Lett. 57, 2999 (1986).

58. J. K. Bhattacharjee, J. Phys. A. 31, L93 (1998).

59. M. Lässig and H. Kinzelbach, Phys. Rev. Lett. 78, 903 (1997).
60. The exponent $\rho$ of the noise correlations must, however, must not be too large. For $\rho>(d+1) / 2$, one enters a regime where $z$ would become less than 1 . In one dimension, Hayot and Jayaprakash 22,23] discuss multi-fractal behavior and intermittency in that regime.

61. T. Hwa and E. Frey, Phys. Rev. A 44, R7873 (1991).

62. E. Frey, U. C. Täuber, and T. Hwa, Phys. Rev. E 53, 4424 (1996).

63. B. Drossel, Phys. Rev. E 54, 2111 (1996).

64. M. Marsili and A. J. Bray, Phys. Rev. Lett. 76, 2750 (1996).

65. T. J. Newman and A. J. Bray, J. Phys. A. 29, 7917 (1996).

66. T. J. Newman and M. R. Swift, Phys. Rev. Lett. 79, 2261 (1997). 